\title{
Quasiprobability distributions in stochastic wave-function methods
}

\author{
Evgeny A. Polyakov" and Pavel N. Vorontsov-Velyaminov ${ }^{\dagger}$ \\ Faculty of Physics, St. Petersburg State University, 198504, St. Petersburg, Russia
}

(Received 12 December 2014; published 8 April 2015)

\begin{abstract}
Quasiprobability distributions emerging in the stochastic wave-function method of Carusotto et al. [Phys. Rev. A 63, 023606 (2001)] are investigated. We show that there are actually two types of quasiprobabilities. The first one, the "diagonal Hartree-Fock state projection" representation, is useful in representing the initial conditions for stochastic simulation in the most compact form. It defines antinormally ordered expansion of the density operator and normally ordered mapping of the observables to be averaged. We completely characterize the equivalence classes of this phase-space representation. The second quasiprobability distribution, the "nondiagonal Hartree-Fock state projection" representation, extends the first one in order to achieve stochastic representation of the quantum dynamics. We demonstrate how the differential identities of the stochastic ansatz generate the automorphisms of this phase-space representation. These automorphisms turn the stochastic representation into a gauge theory. The gauge transformations of the quasiprobability master equation are described. In particular, it is the analyticity of the stochastic ansatz that allows one to transform the master equation into the genuine Fokker-Planck equation. We demonstrate how the different variants of the stochastic wave-function method can be constructed, first by choosing a certain optimality criteria or constraints, and then by satisfying these criteria with a suitable choice of gauge. The problem of boundary terms is considered. It is demonstrated that the simple scheme with Fock states of Carusotto et al. is not subjected to this problem.
\end{abstract}

DOI: 10.1103/PhysRevA.91.042107

PACS number(s): 03.65.Db, 05.30.Ch, 02.70.Ss

\section{INTRODUCTION}

The coherent state projection operator basis [1] with its successive generalizations (the nondiagonal coherent state projections in positive $P$ representations [2-4] and Gaussian operators [5-7]) are of considerable importance in the fields of quantum optics and ultracold gases [8-11]. They are used to construct the generalized phase-space representations of quantum dynamics $[5,6,11,12]$. In these representations, the state of the quantum system, the density operator, is represented as a quasiprobability distribution in a certain (abstract) phase space. The average values of the quantum observables are given as expected values of certain classical functions over this quasiprobability. And, most importantly is that the exact quantum evolution is represented as a drift and diffusion in this phase space. These properties made it possible to develop and conduct the exact Monte Carlo simulations of various many-body dynamic phenomena [8-11].

During the past decade, another approach to the stochastic representations has been developed: the stochastic wavefunction method [13-25]. In this case, a certain set of wave functions is selected, such that this set forms an overcomplete basis. The tensor products of all the possible pairs of these wave functions form an overcomplete operator basis in the space of the density operators. Then, supposing that the two wave functions in a given operator basis element are driven by a general diffusion process with jumps, the conditions are found for which the expectations of this stochastic process reproduce the exact quantum dynamics for a given quantum master equation [13-15,20,21,23,25].

\footnotetext{
*Corresponding author: e.a.polyakov@gmail.com

†voron.wgroup@gmail.com
}

Currently, the phase-space approach is limited to the diffusive stochastic representations, but in principle it can be applied to analytical overcomplete operator basis of the general form, provided the action of the operator $\hat{\Psi}^{\dagger}(\mathbf{x}) \hat{\Psi}\left(\mathbf{x}^{\prime}\right)$ is represented by a first-order differential operator. The phasespace approach is useful in the investigation of how the quantum states are represented by the quasiprobabilities, how the automorphisms of such representations are related to the equivalent transformations of the equations of motion, and how the quantum statements are mapped onto $c$-number functions. On the other hand, the stochastic wave-function approach allows us to consider the general stochastic processes with jumps, however, currently they are limited to the tensor product states $[20-22,25]$. The stochastic wave-function approach allows one to treat a non-Markovian dynamics and it offers a somewhat faster way to the simulations since it allows one to directly obtain the class of the exact stochastic processes.

There is an understanding that these two approaches are complementary pictures of the same subject $[4,11,20]$. Even more, it can be argued that such a division into the two groups of methods is similar to what is happening in the classical theory of stochastic processes, where one group consists of indirect, or analytical, methods which deal with distributions and transition probabilities, and which culminate in the differential Chapman-Kolmogorov master equation for distribution $[26,27]$. The other group consists of direct, or probabilistic, methods which are concerned with the random variables and their trajectories, and which culminate in the theory of stochastic differential equations [26].

Despite this understanding of the equivalence of the two approaches, there is still insufficient research on the quasiprobability distributions which emerge in the stochastic wavefunction methods. Except for the existence theorems [22,28], it is not known whether these distributions define some ordered operator expansions for the density operators and how it is 
connected with the ordered mapping of the observables [20]. For example, in the case of $P$ distributions we have an optical equivalence theorem on the reciprocal ordering of the density operator and the observables [1,29-32]. Is this theorem being discarded when we move to the more general stochastic wave-functions methods, or is it generalized somehow? Aside from fundamental aspects, these questions are of practical importance for simulations since we need to represent the initial quantum state of the system in the most compact and efficient form for the Monte Carlo sampling.

As a first step towards filling this gap, we have chosen a particular stochastic wave-function method, namely, the method of Carusotto et al. [13,28]. In this work, we present a comprehensive study of the quasiprobability distributions emerging in this method. In complete analogy with $P$ function $[2,12]$, we find that there are actually two of them. The first one, the "diagonal Hartree-Fock state projection" functional, is useful in representing the known quantum states (e.g., initial conditions) in the most regular or in the most compact form. This distribution is remarkable in the sense that it allows us to represent any density operator of the $N$-particle bosonic system as a functional in the one-particle Hilbert space. As it turns out, this distribution has the same class of operator ordering representation as the $P$ function [4]: the $P$ function is a realization of the normal ordering mapping for the quantum systems with fixed phase and amplitude, whereas the quasiprobability distribution of Carusotto et al. $[13,28]$ is a realization of the same ordering class for the systems with fixed number of particles.

The second quasiprobability distribution, the "nondiagonal Hartree-Fock state projection" functional, is useful for deriving the stochastic equations, due to its gauge symmetries [12]. More specifically, we demonstrate that the stochastic representation of Carusotto et al. [13,28] is possible due to the analyticity of the Hartree-Fock dyadic ansatz. The optimization of the noise (so that it is orthogonal to the mean-field evolution $[20,21,25])$ is possible due to a special "absorption" differential identity of the Hartree-Fock dyadic ansatz. It is interesting to note that the method of optimal observable evolution [20], which, at first glance, is derived from entirely different considerations, is simply obtained by the choice of such a gauge which conserves the trace of the Hartree-Fock dyadic ansatz.

In Sec. II, we introduce the Hartree-Fock state projection basis. The Wick theorem for the integration over the functional unit sphere is proven in Sec. II A. We use this theorem to prove the overcompleteness of the diagonal Hartree-Fock state projection operators in Sec. II B. In Sec. III, we introduce the diagonal Hartree-Fock state projection representation. In Sec. III A, the Wick theorem is employed to show that any number-conserving $N$-particle density operator in the antinormally ordered form is mapped to the multilinear functionals on the unit sphere. The representation of a given density operator by diagonal Hartree-Fock state projection functional is not unique. The equivalence classes of this quasiprobability distribution are completely characterized in Sec. III B. In Sec. III D, we derive the operator mappings for the master equation. This equation in general does not admit the stochastic interpretation, and in Sec. IV we extend the representation to the nondiagonal Hartree-Fock state projection representation. In Sec. IV B, it is demonstrated that the nonuniqueness of this representation is described by the semigroup of automorphisms, which is generated by the differential identities for the Hartree-Fock dyadic ansatz. These automorphisms turn this phase-space representation into a gauge theory. In Sec. IV C, we use a particular choice of gauge in order to transform the master equation into a geniune Fokker-Planck equation. In Sec. IV D, we describe the gauge transformations that follow from the homogeneity of the Hartree-Fock state (the "absorption property"). We demonstrate how different stochastic methods can be constructed by meeting various optimality criteria or constraints with a suitable choice of gauge. In Sec. V, we discuss the problem of boundary terms and prove that the simple scheme with Fock states of Carusotto et al. [13] is free from this problem. In Appendix A, we reveal the connections with the theory of harmonic multivariate polynomials [33,34], and derive the Wick theorem. Fokker-Planck equations for complex Ito processes [35] have a general form which is reviewed in Appendix B. This general form allows us to easily use the overcomplete basis analyticity in order to bring the master equation into a form which admits the stochastic interpretation.

We believe that the mathematical approaches that we develop in this work will be helpful to study the quasiprobability distributions which emerge in the other stochastic wave-function methods [14,15,17-20,25].

\section{COMPLETENESS OF THE BOSONIC HARTREE-FOCK PROJECTIONS}

The development of the generalized phase-space representations, such as the positive- $P[2,4,12]$ and the Gaussian operator [5-7] representations, were motivated by an attempt to extend the Glauber-Sudarshan $P$ representation [1] in order to make the quasiprobability distribution a genuine probability with stochastic evolution $[2,4,12]$. The operator representations we study in this work are also motivated by stochastic representation of quantum dynamics, but with the Hartree-Fock state projections instead of the coherent ones. This stochastic representation was first introduced in the works of Carusotto et al. [13,28].

Starting from the seminal works of Glauber [29,30], the investigation of every new (generalized) phase-space representation begins with the introduction and treatment of a certain overcomplete operator basis. Our case is not an exception to this direction. In this section, we study the properties of the nondiagonal bosonic Hartree-Fock state projections $[13,28]$

$$
\widehat{\Lambda}\left(\phi, \phi^{+*}\right)=|N: \phi\rangle\left\langle N: \phi^{+}\right|,
$$

where $|N: \phi\rangle=\left[\int d^{n} \mathbf{x} \phi(\mathbf{x}) \hat{\Psi}^{\dagger}(\mathbf{x})\right]^{N}|0\rangle / \sqrt{N !}$ is the Fock state with $N$ particles in one orbital $\phi(\mathbf{x})$ in the $n$-dimensional coordinate space $\mathbb{R}^{n}$. The creation $\hat{\Psi}^{\dagger}(\mathbf{x})$ and annihilation $\hat{\Psi}(\mathbf{x})$ operators obey the standard commutation relations

$$
\left[\hat{\Psi}(\mathbf{x}), \hat{\Psi}^{\dagger}\left(\mathbf{x}^{\prime}\right)\right]=\delta\left(\mathbf{x}-\mathbf{x}^{\prime}\right) .
$$

In the works of Carusotto et al. [13,28], the following properties of the operator basis (1) were established: (i) the resolution of unity in the $N$-particle subspace of the Fock 
space

$$
P_{N} \propto \int_{\|\phi\|=1} \mathcal{D} \phi \mathcal{D} \phi^{*}|N: \phi\rangle\langle N: \phi|,
$$

where $P_{N}$ is the projection of the Fock space onto the $N$ particle subspace. The integration is done over the unit sphere in the functional (Hilbert) space $H$ of one-particle orbitals $\phi$. (ii) Each number-conserving density operator in the $N$-particle subspace can be expanded over the basis (1):

$$
\begin{aligned}
P_{N} \hat{\rho} P_{N} \propto & \int \mathcal{D} \phi \mathcal{D} \phi^{*} \mathcal{D} \phi^{+} \mathcal{D} \phi^{+*} \\
& \times \mathcal{P}\left(\phi, \phi^{*}, \phi^{+}, \phi^{+*}\right)|N: \phi\rangle\left\langle N: \phi^{+}\right|,
\end{aligned}
$$

and the expansion coefficient $\mathcal{P}\left(\phi, \phi^{*}, \phi^{+}, \phi^{+*}\right)$ is not unique and can be chosen positive.

In the works of Carusotto et al. [13,28], the abovementioned properties were established indirectly through the use of coherent states. Following in this section we derive the expansions (3) and (4) using a generic method which will allow us to reveal the (generalized) phase-space structure and derive explicit formulas for $\mathcal{P}\left(\phi, \phi^{*}, \phi^{+}, \phi^{+*}\right)$.

\section{A. Wick theorem for functional integration over a unit sphere}

Expanding the definition of the Hartree-Fock states $|N: \phi\rangle$ in Eqs. (3) and (4), one can note that it all comes down to the moments of a unit sphere,

$$
\begin{aligned}
& I\left(\mathbf{x}_{1}, \ldots, \mathbf{x}_{p}, \mathbf{y}_{1}, \ldots, \mathbf{y}_{q}\right) \\
& \quad=\int_{\|\phi\|=1} \mathcal{D} \phi \mathcal{D} \phi^{*} \phi\left(\mathbf{x}_{1}\right) \ldots \phi\left(\mathbf{x}_{p}\right) \phi^{*}\left(\mathbf{y}_{1}\right) \ldots \phi^{*}\left(\mathbf{y}_{q}\right) .
\end{aligned}
$$

We define the functional integral (5) as a limit of integrals in the finite-dimensional complex Euclidean space $\mathbb{C}^{\mathcal{N}}$ when the dimension $\mathcal{N}$ tends to $\infty$. The relevant space $\mathbb{C}^{\mathcal{N}}$ is obtained through restriction of the one-particle coordinate space $\mathbb{R}^{n}$ to a finite volume $V$ with appropriate boundary conditions, and by introducing a lattice of sites $\mathbf{x}_{k}, k=1, \ldots, \mathcal{N}$, such that each lattice site occupies the volume $\Delta V=V / \mathcal{N}$. The space $\mathbb{C}^{\mathcal{N}}$ is comprised of all the vectors $\phi=\left[\phi\left(\mathbf{x}_{1}\right), \ldots, \phi\left(\mathbf{x}_{\mathcal{N}}\right)\right]$. Until now, the lattice $\mathbf{x}_{k}$ is arbitrary. However, in order to establish the connection between the space $\mathbb{C}^{\mathcal{N}}$ and the original one-body Hilbert space $H$, we assume that the lattice allows us to define the orthonormal orbitals $\delta_{\mathcal{N}}(k), k=1, \ldots, \mathcal{N}$, with the following properties. Each orbital $\delta_{\mathcal{N}}(k)$ is associated with the lattice site $\mathbf{x}_{k}$. By association we mean the site-orbital orthogonality condition: for any $k$ and $l$ we have $\left\langle\mathbf{x}_{k} \mid \delta_{\mathcal{N}}(l).\right\rangle=$ $\Delta V^{-1 / 2} \delta_{k l}$. Each orbital $\delta_{\mathcal{N}}(k)$ adheres to the volume $V$ boundary conditions. These orbitals span the $\mathcal{N}$-dimensional subspace $H_{\mathcal{N}}$ of the Hilbert space $H$. The subspace $H_{\mathcal{N}}$ consists of the orbitals $\phi(\mathbf{x})$ which are completely determined by their values on the lattice sites $\mathbf{x}_{k}$ and which adhere to the volume $V$ boundary conditions. Moreover, the following properties follow from our definitions:

$$
\phi\left(\mathbf{x}_{k}\right)=\frac{1}{\sqrt{\Delta V}}\left\langle\phi \mid \delta_{\mathcal{N}}(k)\right\rangle
$$

and

$$
\phi(\mathbf{x})=\sqrt{\Delta V} \sum_{k} \phi\left(\mathbf{x}_{k}\right)\left\langle\mathbf{x} \mid \delta_{\mathcal{N}}(k)\right\rangle .
$$

Then, the inner product for any two orbitals $\phi$ and $\varphi$ from $H_{\mathcal{N}}$ assumes the form

$$
\langle\phi \mid \varphi\rangle=\Delta V \sum_{k} \phi^{*}\left(\mathbf{x}_{k}\right) \varphi\left(\mathbf{x}_{k}\right)=:\langle\boldsymbol{\phi} \mid \boldsymbol{\varphi}\rangle,
$$

where hereinafter by bold letters we denote the vectors from $\mathbb{C}^{\mathcal{N}}$. The relation $(8)$ means that the spaces $\mathbb{C}^{\mathcal{N}}$ and $H^{\mathcal{N}}$ are isometric to each other. We can say that the space $\mathbb{C}^{\mathcal{N}}$ is the lattice picture of the Hilbert subspace $H_{\mathcal{N}}$. The approximation we make during the lattice discretization is the projection of the full Hilbert space $H$ onto $H_{\mathcal{N}}$. All these observations provide us with a rigorous way to convert the lattice sums into the integrals, $\int d^{n} \mathbf{x}=\Delta V \sum_{k}$.

The unit vectors $\|\phi\|=1$ in $H$ correspond to $\|\boldsymbol{\phi}\|=$ $1 / \sqrt{\Delta V}$ in $\mathbb{C}^{\mathcal{N}}$, and in the lattice picture the integral (5) is

$$
\begin{aligned}
& I\left(r_{1}, \ldots, r_{p}, s_{1}, \ldots, s_{q}\right) \\
& \quad=\int_{\|\boldsymbol{\phi}\|=1 / \sqrt{\Delta V}} \prod_{k=1}^{\mathcal{N}} d \phi_{k} d \phi_{k}^{*} \phi_{r_{1}} \ldots \phi_{r_{p}} \phi_{s_{1}}^{*} \ldots \phi_{s_{q}}^{*},
\end{aligned}
$$

where $\phi_{k}=\phi\left(\mathbf{x}_{k}\right)$. In Appendix A, we employ the theory of harmonic polynomials $[33,34]$ to evaluate this integral:

$$
\begin{aligned}
& I\left(r_{1}, \ldots, r_{p}, s_{1}, \ldots, s_{q}\right) \\
& \quad=\delta_{p, q} S_{2 \mathcal{N}-1} \frac{(\mathcal{N}-1) !}{(\mathcal{N}-1+p) !} \sum_{P} \prod_{t=1}^{p} \frac{1}{\Delta V} \delta_{r_{t}, s_{P(t)}},
\end{aligned}
$$

where the sum over $P$ is the sum over all the permutations $P$ of the numbers $1, \ldots, p$, and $S_{2 \mathcal{N}-1}$ is the volume of the sphere in $\mathbb{C}^{\mathcal{N}}$ :

$$
\begin{aligned}
S_{2 \mathcal{N}-1} & =\int_{\|\phi\|=1 / \sqrt{\Delta V}} \prod_{k=1}^{\mathcal{N}} d \phi_{k} d \phi_{k}^{*} \\
& =\frac{2(2 \pi)^{\mathcal{N}}}{\Delta V^{\mathcal{N}-1 / 2}} \frac{1}{(\mathcal{N}-1) !} .
\end{aligned}
$$

The relation (10) has the form of the Wick theorem: the value of the integral is the sum of all the possible pairings between the $\phi$ sites and the $\phi^{*}$ sites, and each pair is connected with the delta propagator multiplied by a constant normalization factor

$$
\Gamma_{\mathcal{N}}(p)=S_{2 \mathcal{N}-1} \frac{(\mathcal{N}-1) !}{(\mathcal{N}-1+p) !} .
$$

\section{B. Completeness of the Hartree-Fock state projections}

We introduce the annihilation operators at the lattice sites $\mathbf{x}_{k}$ as

$$
\widehat{a}_{k}=\frac{1}{\sqrt{\Delta V}} \int d^{n} \mathbf{x}\left\langle\delta_{\mathcal{N}}(k) \mid \mathbf{x}\right\rangle \widehat{\Psi}(\mathbf{x})
$$

with the commutation relations

$$
\left[\widehat{a}_{k}, \widehat{a}_{k^{\prime}}^{\dagger}\right]=\frac{\delta_{k, k^{\prime}}}{\Delta V}
$$

The lattice Fock state is defined as $|N: \boldsymbol{\phi}\rangle=$ $\left[\Delta V \sum_{k} \phi_{k} \widehat{a}_{k}^{\dagger}\right]^{N}|0\rangle / \sqrt{N !}$. Now, we derive the resolution of unity (3). We consider the right-hand side of (3) in the lattice 
picture and apply to it the Wick theorem (10):

$$
\begin{aligned}
\int_{\|\boldsymbol{\phi}\|=1 / \sqrt{\Delta V}} \prod_{k=1}^{\mathcal{N}} d \phi_{k} d \phi_{k}^{*}|N: \boldsymbol{\phi}\rangle\langle N: \boldsymbol{\phi}| \\
=S_{2 \mathcal{N}-1} \frac{(\mathcal{N}-1) !}{(\mathcal{N}-1+N) !} \Delta V^{N} \\
\quad \times \sum_{k_{1}, \ldots k_{N}} \widehat{a}_{k_{1}}^{\dagger} \ldots \widehat{a}_{k_{N}}^{\dagger}|0\rangle\langle 0| \widehat{a}_{k_{1}} \ldots \widehat{a}_{k_{N}} \\
=S_{2 \mathcal{N}-1} \frac{(\mathcal{N}-1) !}{(\mathcal{N}-1+N) !} N ! \Delta V^{N} \\
\quad \times \sum_{k_{1}, \ldots k_{N}}^{\prime}\left|\mathbf{x}_{k_{1}}, \ldots, \mathbf{x}_{k_{N}}\right\rangle\left\langle\mathbf{x}_{k_{1}}, \ldots, \mathbf{x}_{k_{N}}\right|
\end{aligned}
$$

where in the last line the symbol $\sum^{\prime}$ denotes the sum over all the ordered $N$-tuples $k_{1}, \ldots, k_{N}$, i.e., we do not count twice the tuples which differ only by permutation of values. The state $\left|\mathbf{x}_{k_{1}}, \ldots, \mathbf{x}_{k_{N}}\right\rangle$ in the lattice picture corresponds to the particles occupying the orbitals $\delta_{\mathcal{N}}\left(k_{1}\right), \ldots, \delta_{\mathcal{N}}\left(k_{N}\right)$ in the subspace $H^{\mathcal{N}}$. Therefore, the states $\left|\mathbf{x}_{k_{1}}, \ldots, \mathbf{x}_{k_{N}}\right\rangle$ form the complete basis in the $N$-particle subspace of the Fock space over $H^{\mathcal{N}}$. With this in mind, we finish the derivation (15) of the completeness relation (3) as

$$
\begin{aligned}
P_{N}= & \frac{(\mathcal{N}-1+N) !}{S_{2 \mathcal{N}-1} N !(\mathcal{N}-1) !} \\
& \times \int_{\|\boldsymbol{\phi}\|=1 / \sqrt{\Delta V}} \prod_{k=1}^{\mathcal{N}} d \phi_{k} d \phi_{k}^{*}|N: \boldsymbol{\phi}\rangle\langle N: \boldsymbol{\phi}| .
\end{aligned}
$$

Note that the similar result in the work [28] lacks the factor $(\Delta V)^{\mathcal{N}-1 / 2}$. The relation (16) is an analog of the completeness property of the coherent states in the Glauber-Sudarshan $P$ representation [1].

\section{DIAGONAL HARTREE-FOCK STATE PROJECTION REPRESENTATION}

Continuing the analogy with the Glauber-Sudarshan $P$ representation [1], further we introduce and study the diagonal Hartree-Fock state projection representation of the density operators

$$
P_{N} \hat{\rho} P_{N}=\int_{\|\boldsymbol{\phi}\|=1 / \sqrt{\Delta V}} \prod_{k=1}^{\mathcal{N}} d \phi_{k} d \phi_{k}^{*} \mathcal{P}\left(\boldsymbol{\phi}, \boldsymbol{\phi}^{*}\right)|N: \boldsymbol{\phi}\rangle\langle N: \boldsymbol{\phi}| .
$$

\section{A. Operator mappings for the density operator}

We will derive the relation between $\hat{\rho}$ and $\mathcal{P}\left(\boldsymbol{\phi}, \boldsymbol{\phi}^{*}\right)$. It is natural to begin the treatment by assuming that $\mathcal{P}\left(\boldsymbol{\phi}, \boldsymbol{\phi}^{*}\right)$ can be represented as a convergent series of homogeneous polynomials in $\boldsymbol{\phi}$ and $\boldsymbol{\phi}^{*}$. Due to the Wick theorem (10), only the terms with equal powers of $\boldsymbol{\phi}$ and $\boldsymbol{\phi}^{*}$ need to be considered. So, we have

$$
\mathcal{P}\left(\boldsymbol{\phi}, \boldsymbol{\phi}^{*}\right)=\sum_{m=0}^{\infty} \mathcal{P}_{(m, m)}\left(\boldsymbol{\phi}, \boldsymbol{\phi}^{*}\right),
$$

where the $(m, m)$-multilinear form $\mathcal{P}_{(m, m)}\left(\boldsymbol{\phi}, \boldsymbol{\phi}^{*}\right)$ is defined according to (A3) in Appendix A. Substituting the expansion (18) into the right-hand side of (17) and evaluating it term by term, we obtain that any operator $\hat{\rho}$, which is representable by (18) and (17), can be expanded as

$$
P_{N} \hat{\rho} P_{N}=\sum_{m=0}^{\infty} P_{N} \hat{\rho}_{(m, m)} P_{N} .
$$

To evaluate the term $\hat{\rho}_{(m, m)}$ we substitute the expression (A3) for the form $\mathcal{P}_{(m, m)}$ into the right-hand side of (17) and use the Wick theorem (10) obtaining the following result:

$$
\begin{aligned}
P_{N} \hat{\rho}_{(m, m)} P_{N}= & \Gamma_{\mathcal{N}}(m+N) \sum_{j=\max (0, m-N)}^{m} \frac{m !^{2} N !}{j !(m-j) !^{2}(N-m+j) !} \prod_{r=1}^{m}\left[\Delta V \sum_{k_{r}=1}^{\mathcal{N}}\right] \prod_{s=j+1}^{m}\left[\Delta V \sum_{k_{s}^{\prime}=1}^{\mathcal{N}}\right] \\
& \times h\left(\mathbf{x}_{k_{1}}, \ldots, \mathbf{x}_{k_{j}}, \mathbf{x}_{k_{j+1}}, \ldots, \mathbf{x}_{k_{m}} \mid \mathbf{x}_{k_{1}}, \ldots, \mathbf{x}_{k_{j}}, \mathbf{x}_{k_{j+1}^{\prime}}, \ldots, \mathbf{x}_{k_{m}^{\prime}}\right) \widehat{a}_{k_{j+1}}^{\dagger} \ldots \widehat{a}_{k_{m}}^{\dagger} \\
& \times\left\{\prod_{t=1}^{N-m+j}\left[\Delta V \sum_{k_{t}^{\prime \prime}=1}^{\mathcal{N}}\right] \widehat{a}_{k_{1}^{\prime \prime}}^{\dagger} \ldots \widehat{a}_{k_{N-m+j}^{\prime \prime}}^{\dagger}|0\rangle\langle 0| \widehat{a}_{k_{1}^{\prime \prime}} \ldots \widehat{a}_{k_{N-m+j}^{\prime \prime}}\right\} \widehat{a}_{k_{j+1}^{\prime}} \ldots \widehat{a}_{k_{m}^{\prime}} .
\end{aligned}
$$

Here, $h$ is the tensor of the form $\mathcal{P}_{(m, m)}$; each term in the sum over $j$ represents the situation when $j$ pairings are inside $\mathcal{P}_{(m, m)}\left(\boldsymbol{\phi}, \boldsymbol{\phi}^{*}\right)$ and $N-m+j$ pairings are inside $|N: \boldsymbol{\phi}\rangle\langle N: \boldsymbol{\phi}|$. The remaining $m-j$ pairings are between $\mathcal{P}_{(m, m)}\left(\boldsymbol{\phi}, \boldsymbol{\phi}^{*}\right)$ and $|N: \boldsymbol{\phi}\rangle\langle N: \boldsymbol{\phi}|$. The combinatorial prefactor is the number of ways for such a partitioning of the pairings, divided by the Fock state normalization $N$ !. We note that the expression in the curly braces in (20) is the unnormalized projection $(N-m+j) ! P_{N-m+j}$ into the $(N-m+j)$-particle Fock subspace. Therefore, using the identity

$$
P_{N} \widehat{a}_{k_{1}}^{\dagger} \ldots \widehat{a}_{k_{l}}^{\dagger} \widehat{a}_{k_{1}^{\prime}} \ldots \widehat{a}_{k_{l}^{\prime}} P_{N}=\widehat{a}_{k_{1}}^{\dagger} \ldots \widehat{a}_{k_{l}}^{\dagger} P_{N-l} \widehat{a}_{k_{1}^{\prime}} \ldots \widehat{a}_{k_{l}^{\prime}},
$$

we rewrite the result (20) as

$$
\begin{aligned}
P_{N} \hat{\rho}_{(m, m)} P_{N}= & P_{N} \Gamma_{\mathcal{N}}(m+N) \sum_{j=\max (0, m-N)}^{m} \frac{m !^{2} N !}{j !(m-j) !^{2}} \prod_{r=1}^{m}\left[\Delta V \sum_{k_{r}=1}^{\mathcal{N}}\right] \prod_{s=j+1}^{m}\left[\Delta V \sum_{k_{s}^{\prime}=1}^{\mathcal{N}}\right] \\
& \times h\left(\mathbf{x}_{k_{1}}, \ldots, \mathbf{x}_{k_{j}}, \mathbf{x}_{k_{j+1}}, \ldots, \mathbf{x}_{k_{m}} \mid \mathbf{x}_{k_{1}}, \ldots, \mathbf{x}_{k_{j}}, \mathbf{x}_{k_{j+1}^{\prime}}, \ldots, \mathbf{x}_{k_{m}^{\prime}}\right) \widehat{a}_{k_{j+1}}^{\dagger} \ldots \widehat{a}_{k_{m}}^{\dagger} \widehat{a}_{k_{j+1}^{\prime}} \ldots \widehat{a}_{k_{m}^{\prime}} P_{N} .
\end{aligned}
$$


Here, the expression on the right-hand side is a normally ordered form of the following antinormally ordered operator:

$$
\begin{aligned}
P_{N} \hat{\rho}_{(m, m)} P_{N}= & P_{N} \Gamma_{\mathcal{N}}(m+N) N ! \\
& \times \prod_{r=1}^{m}\left[\Delta V \sum_{k_{r}=1}^{\mathcal{N}} \widehat{a}_{k_{r}}\right] \prod_{s=1}^{m}\left[\Delta V \sum_{k_{s}^{\prime}=1}^{\mathcal{N}} \widehat{a}_{k_{s}^{\prime}}^{\dagger}\right] \\
& \times h\left(\mathbf{x}_{k_{1}}, \ldots, \mathbf{x}_{k_{m}} \mid \mathbf{x}_{k_{1}^{\prime}}, \ldots, \mathbf{x}_{k_{m}^{\prime}}\right) P_{N},
\end{aligned}
$$

that can be shown directly by bringing the operator in the right-hand side of (23) into the normal form.

We have obtained that (i) the general $(m, m)$-multilinear form $\mathcal{P}_{(m, m)}\left(\boldsymbol{\phi}, \boldsymbol{\phi}^{*}\right)$ with tensor $h$ corresponds to a general projected antinormal $m$-particle operator (23); (ii) any numberconserving physical density operator $\hat{\rho}$ in the $N$-particle subspace of the Fock space can be mapped to the corresponding $\mathcal{P}\left(\boldsymbol{\phi}, \boldsymbol{\phi}^{*}\right)$ by putting $\hat{\rho}$ into the antinormally ordered form and using the mapping rule

$$
\begin{aligned}
& P_{N} \widehat{a}_{k_{1}} \ldots \widehat{a}_{k_{m}} \widehat{a}_{k_{1}^{\prime}}^{\dagger} \ldots \widehat{a}_{k_{m}^{\prime}}^{\dagger} P_{N} \\
& \quad \rightarrow \frac{1}{\Gamma_{\mathcal{N}}(m+N) N !} \phi_{k_{1}}^{*} \ldots \phi_{k_{m}}^{*} \phi_{k_{1}^{\prime}} \ldots \phi_{k_{m}^{\prime}} .
\end{aligned}
$$

The discretized integrals in the expression (A3) for $\mathcal{P}_{(m, m)}\left(\boldsymbol{\phi}, \boldsymbol{\phi}^{*}\right)$ are converging as $\mathcal{N} \rightarrow \infty$ since for any physical density operator $\langle\phi, \ldots, \phi|\hat{\rho}| \phi, \ldots, \phi\rangle<\infty$. Hence, the number-conserving physical density operators $\hat{\rho}$ in the $N$ particle subspace can be represented by polynomials of the order $2 N$ on the unit sphere.

\section{B. Nonuniqueness of the diagonal Hartree-Fock state projection representation}

We call the two functionals $\mathcal{P}\left(\boldsymbol{\phi}, \boldsymbol{\phi}^{*}\right)$ and $\mathcal{P}^{\prime}\left(\boldsymbol{\phi}, \boldsymbol{\phi}^{*}\right)$ equivalent if they correspond to the same operator $P_{N} \hat{\rho} P_{N}$ in the expansion (17). Therefore, each operator $P_{N} \hat{\rho} P_{N}$ defines an equivalence class in the space of functionals $\mathcal{P}\left(\boldsymbol{\phi}, \boldsymbol{\phi}^{*}\right)$. In this section, we derive the complete characterization of such equivalence classes.

The basis $\widehat{\Lambda}\left(\phi, \phi^{*}\right)$ is overcomplete. This means that there is a space $Z$ of quasiprobability functionals $\mathcal{Z}\left(\boldsymbol{\phi}, \boldsymbol{\phi}^{*}\right)$ such that

$$
\int d \Omega \mathcal{Z}\left(\boldsymbol{\phi}, \boldsymbol{\phi}^{*}\right) \widehat{\Lambda}\left(\phi, \phi^{*}\right)=0,
$$

where the element of the generalized solid angle $d \Omega$ is defined in (A8). We call $Z$ the "zero representation" space, since in the expansion (17) it corresponds to $P_{N} \hat{\rho} P_{N}=0$. Let us define the space $D$ consisting of all the quasiprobability functionals with finite $L^{2}$ norm on the unit sphere. The space $D$ can be represented as the direct sum

$$
D=R \oplus Z,
$$

where the space $R$ is the orthogonal complement to $Z$, $R=Z^{\perp}$, and we call $R$ the minimal representation space, because every $\mathcal{P}\left(\boldsymbol{\phi}, \boldsymbol{\phi}^{*}\right)$ from $R$ corresponds to a certain nonzero $P_{N} \hat{\rho} P_{N}$ in (17), and such $\mathcal{P}\left(\boldsymbol{\phi}, \boldsymbol{\phi}^{*}\right)$ has the property of having the lowest $L^{2}$ norm among all the functionals from the same equivalence class. These considerations lead us to the conclusion that there exists one-to-one correspondence between the equivalence classes and the elements in $R$, and that all the nonuniqueness is described by the space $Z$. Following, we find the spaces $R$ and $Z$ for the diagonal representation (17).

The representation of functional $\mathcal{P}\left(\boldsymbol{\phi}, \boldsymbol{\phi}^{*}\right)$ by the series (18) is not unique since the diagonal expansion (17) is defined on the unit sphere, and hence each of the terms in the series (18) can be multiplied by the factor

$$
\Delta V \sum_{k=1}^{\mathcal{N}} \phi_{k}^{*} \phi_{k}=1
$$

i.e., we can formally change the powers of the terms $\mathcal{P}_{(m, m)}\left(\boldsymbol{\phi}, \boldsymbol{\phi}^{*}\right)$ without changing the value of $\mathcal{P}\left(\boldsymbol{\phi}, \boldsymbol{\phi}^{*}\right)$. To get rid of this ambiguity, we decompose each term $\mathcal{P}_{(m, m)}\left(\boldsymbol{\phi}, \boldsymbol{\phi}^{*}\right)$ into the harmonic multilinear forms (see Appendix A), and obtain the series expansion

$$
\mathcal{P}\left(\boldsymbol{\phi}, \boldsymbol{\phi}^{*}\right)=\sum_{m=0}^{\infty} \mathcal{P}_{(m, m)}^{(0)}\left(\boldsymbol{\phi}, \boldsymbol{\phi}^{*}\right),
$$

where each term $\mathcal{P}_{(m, m)}^{(0)}$ is now harmonic and its tensor $h_{(m, m)}$ is traceless, $\operatorname{Tr}_{1} h_{(m, m)}=0$. According to Appendix A, each functional $\mathcal{P}\left(\boldsymbol{\phi}, \boldsymbol{\phi}^{*}\right)$ is uniquely represented by the expansion (28).

Now, let us find the operator $P_{N} \hat{\rho}_{(m, m)} P_{N}$ which corresponds to the harmonic form $\mathcal{P}_{(m, m)}^{(0)}$. We substitute its tensor $h_{(m, m)}$ into the result (22), and obtain the phase-space correspondence relations

$$
\begin{aligned}
\mathcal{P}_{(m, m)}^{(0)}\left(\boldsymbol{\phi}, \boldsymbol{\phi}^{*}\right) \longleftrightarrow & P_{N} \Gamma_{\mathcal{N}}(m+N) N ! \\
& \times \prod_{r=1}^{m}\left[\Delta V \sum_{k_{r}=1}^{\mathcal{N}} \widehat{a}_{k_{r}}^{\dagger}\right] \prod_{s=1}^{m}\left[\Delta V \sum_{k_{s}^{\prime}=1}^{\mathcal{N}} \widehat{a}_{k_{s}^{\prime}}\right] \\
& \times h_{(m, m)}\left(\mathbf{x}_{k_{1}}, \ldots, \mathbf{x}_{k_{j}} \mid \mathbf{x}_{k_{1}^{\prime}}, \ldots, \mathbf{x}_{k_{j}^{\prime}}\right) P_{N}
\end{aligned}
$$

for $m \leq N$, and

$$
\mathcal{P}_{(m, m)}^{(0)}\left(\boldsymbol{\phi}, \boldsymbol{\phi}^{*}\right) \longleftrightarrow 0
$$

for $m>N$. Here, in deriving (29) and (30) we use the fact that for the harmonic form $\mathcal{P}_{(m, m)}^{(0)}$ only the situation with zero pairings $(j=0)$ inside $\mathcal{P}_{(m, m)}^{(0)}$ is realized in (22). Due to orthogonality of harmonic forms $\mathcal{P}_{(m, m)}^{(0)}$ with different $m$ (see Appendix A), we conclude that the minimal representation space $R$ consists of all the harmonic forms $\mathcal{P}_{(m, m)}^{(0)}$ of the order $m \leq N$. The phase-space mapping is normally ordered (29) provided we put the number-conserving density operator into the normally ordered form with traceless tensors. The zero representation space $Z$ consists of all the multilinear harmonic forms $\mathcal{P}_{(r, s)}^{(0)}$ with $r \neq s$, and of all the harmonic forms $\mathcal{P}_{(m, m)}^{(0)}$ with $m>N$.

The series (18) is actually a hyperspherical harmonics expansion, which is defined and is converging for any tempered distribution $\mathcal{P}\left(\boldsymbol{\phi}, \boldsymbol{\phi}^{*}\right)$ on the unit sphere. Therefore, any tempered distribution $\mathcal{P}\left(\boldsymbol{\phi}, \boldsymbol{\phi}^{*}\right)$ can be mapped to a numberconserving $N$-particle operator provided the series (19) are converging. The equivalence class of such tempered distributions consists of all such functionals $\mathcal{P}\left(\boldsymbol{\phi}, \boldsymbol{\phi}^{*}\right)$ which, after being decomposed into the harmonic forms of type $(m, m)$, have the first $N+1$ terms equal. 


\section{Compact representations}

Suppose that we have chosen a certain orthonormal basis $\psi_{l}$ in the space $\mathbb{C}^{\mathcal{N}}$ in a physically motivated way. For example, if our aim is to simulate the Bose-Einstein condensation phenomena, we can choose $\psi_{0}$ as the Gross-Pitaevskii state, and $\psi_{1}, \ldots, \psi_{\mathcal{N}}$ as one-particle excitations of increasing energy. Then, if we consider the matrix elements of the density operator with respect to this basis, the element $\langle N$ : $\left.\boldsymbol{\psi}_{0}|\hat{\rho}| N: \boldsymbol{\psi}_{0}\right\rangle$ has the largest weight, and as we admix the excited orbitals $\psi_{l}$ into this matrix element, the weight of the matrix element is rapidly decreasing. In this situation, it is highly inefficient to sample the quasiprobability from the whole functional unit sphere in the expansion (17). We suggest to group together the matrix elements containing the same set of orbitals $K=\left\{\psi_{K_{1}}, \ldots, \psi_{K_{p}}\right\}$ in its bra and ket sides. This is equivalent to splitting of the density operator $\hat{\rho}$ as

$$
\hat{\rho}=\sum_{K} \sum_{K^{\prime} \cup K^{\prime \prime}=K} P_{K^{\prime}} \hat{\rho} P_{K^{\prime \prime}}=: \sum_{K} \llbracket \hat{\rho} \rrbracket_{K},
$$

where the sum over $K$ is the sum over all the different sets of orbitals; the sum over $K^{\prime}$ and $K^{\prime \prime}$ is the sum over all the partitionings of the set $K$ into two (possibly overlapping) parts $K^{\prime}$ and $K^{\prime \prime} ; P_{K^{\prime}}$ is the projector onto the space spanned by all such $N$-particle states that each orbital in the set $K^{\prime}$ is occupied by at least one particle, and there are no occupied orbitals which are not from this set. For each set $K$ consisting of $p$ orbitals we introduce the unit sphere $S_{2 p-1}^{(K)}$ in the space $H_{K}$ spanned by the vectors $\psi_{K_{1}}, \ldots, \boldsymbol{\psi}_{K_{p}}$. We consider the diagonal Hartree-Fock state projection expansion on $S_{2 p-1}^{(K)}$ :

$$
\begin{aligned}
P_{N} \llbracket \hat{\rho} \rrbracket_{K} P_{N}= & \int_{S_{2 p-1}^{(K)}} d \varphi_{1} d \varphi_{1}^{*} \ldots d \varphi_{p} d \varphi_{p}^{*} \\
& \times \mathcal{P}_{K}\left(\boldsymbol{\varphi}, \boldsymbol{\varphi}^{*}\right)|N: \boldsymbol{\varphi}\rangle\langle N: \boldsymbol{\varphi}|,
\end{aligned}
$$

where the vector $\varphi$ is the representation of the wave functions from $H_{K}$ in the picture $\psi_{K_{1}}, \ldots, \psi_{K_{p}}$. Applying the same techniques as in previous sections, we find that the distribution $\mathcal{P}_{K}\left(\varphi, \varphi^{*}\right)$ exists for any bounded number-conserving operator $\llbracket \hat{\rho} \rrbracket_{K}$, it has the same uniqueness properties and the same correspondence relations (24), (29), and (30), as in the case of the diagonal expansion (17), except that the normalization factor $\Gamma_{\mathcal{N}}(m+N)$ is substituted by

$$
\Gamma_{\mathcal{N}}(m+N) \rightarrow \Gamma_{p}(m+N),
$$

and we should set $\Delta V=1$ everywhere. In the practical algorithm, we can sample the initial conditions via random walk with the steps of two kinds. The step of the first type (more frequent) is to sample the initial conditions from the quasiprobability distribution for a given $K$ according to (32). We can make this quasiprobability distribution positive using the trick described at the beginning of Sec. IV [Eqs. (55) and (56)]. The step of the second kind is to change randomly the set $K \rightarrow K^{\prime}$ according to the importance sampling algorithm with the weights $W(K)=\mathcal{P}_{K}\left(\boldsymbol{\varphi}, \boldsymbol{\varphi}^{*}\right)$ and $W\left(K^{\prime}\right)=\mathcal{P}_{K^{\prime}}\left(\boldsymbol{\varphi}, \boldsymbol{\varphi}^{*}\right)$ (or some a priori weight as in [36]). Although, of course, this is only a sketch of the algorithm. The actual practical implementation will reveal all the necessary nuances.

\section{Quasiprobability master equation}

Assume that we have a system of particles in a space of dimension $n$. The particles are in an external potential $V_{\text {ext }}$ and they interact with a pairwise translationally invariant potential $V_{\text {int }}$. The number of particles is exactly $N$. The Hamiltonian of the system in the second quantized form is

$$
\begin{aligned}
\widehat{H}= & \frac{\hbar^{2}}{2 m} \int \nabla \widehat{\Psi}^{\dagger}(\mathbf{x}) \nabla \widehat{\Psi}(\mathbf{x}) d^{n} \mathbf{x} \\
& +\int V_{\text {ext }}(\mathbf{x}) \widehat{\Psi}^{\dagger}(\mathbf{x}) \widehat{\Psi}(\mathbf{x}) d^{n} \mathbf{x} \\
& +\frac{1}{2} \int V_{\mathrm{int}}(\mathbf{x}-\mathbf{y}) \widehat{\Psi}^{\dagger}(\mathbf{x}) \widehat{\Psi}^{\dagger}(\mathbf{y}) \\
& \times \widehat{\Psi}(\mathbf{y}) \widehat{\Psi}(\mathbf{x}) d^{n} \mathbf{x} d^{n} \mathbf{y} .
\end{aligned}
$$

As far as the potential $V_{\text {int }}$ is translationally invariant, later it will be convenient to use the momentum picture. For simplicity, we suppose therefore that the volume $V$ is represented by $n$-dimensional rectangular parallelepiped with the edges of the lengths $L_{1}, \ldots, L_{n}$; hence, $V=\prod_{i=1}^{n} L_{i}$. The lattice is represented by the vectors

$$
\mathbf{x}_{r}=\left(\left(\boldsymbol{n}_{r}\right)_{1} \frac{L_{1}}{\mathcal{N}_{1}}, \ldots,\left(\boldsymbol{n}_{r}\right)_{n} \frac{L_{n}}{\mathcal{N}_{n}}\right)
$$

where $r=1, \ldots, \mathcal{N}$; the index vector $\boldsymbol{n}_{r}$ of the site $r$ is a vector with integer components $-M_{j} \leq\left(\boldsymbol{n}_{r}\right)_{j} \leq M_{j}, M_{j}=\left(\mathcal{N}_{j}-\right.$ 1) $/ 2$ for $j=1, \ldots, n$. The subspace $H_{\mathcal{N}}$ is obtained by the momentum cutoff at $p_{j}^{\max }=2 \pi M_{j} / L_{j}$ along the dimension $j$. Therefore, in the momentum picture the lattice is

$$
\boldsymbol{p}_{r}=2 \pi\left(\frac{\left(\boldsymbol{n}_{r}\right)_{1}}{L_{1}}, \ldots, \frac{\left(\boldsymbol{n}_{r}\right)_{n}}{L_{n}}\right)
$$

In the lattice picture, this Hamiltonian takes on the form

$$
\widehat{H}=\Delta V^{2} \sum_{r, s} h_{r s} \widehat{a}_{r}^{\dagger} \widehat{a}_{s}+\frac{1}{2} \Delta V^{2} \sum_{r, s} V_{r s} \widehat{a}_{r}^{\dagger} \widehat{a}_{s}^{\dagger} \widehat{a}_{s} \widehat{a}_{r}
$$

where $h_{r s}=\left\langle\delta_{\mathcal{N}}(r)|\hat{h}| \delta_{\mathcal{N}}(s)\right\rangle$ contains all the one-body terms and $V_{r s}=\left\langle\delta_{\mathcal{N}}(r) \delta_{\mathcal{N}}(s)\left|\widehat{V}_{\text {int }}\right| \delta_{\mathcal{N}}(s) \delta_{\mathcal{N}}(r)\right\rangle$ is the interaction term.

The state of the system is represented by the density operator $\widehat{\rho}(t)$ acting in the space of Fock states. The exact time evolution of the density operator is governed by the quantum Liouville equation

$$
\partial_{t} \widehat{\rho}(t)=\frac{1}{i \hbar}[\widehat{H}, \widehat{\rho}(t)] .
$$

In the diagonal Hartree-Fock state projection representation (17), the state $\widehat{\rho}(t)$ is represented by a time-dependent functional $\mathcal{P}\left(\boldsymbol{\phi}, \boldsymbol{\phi}^{*}, t\right)$. To find the master equation for $\mathcal{P}$ which is consistent with the quantum Liouville equation (38), we proceed in the same way as it is adopted in the conventional generalized phase-space techniques $[2,5,6,12]$. We start by deriving the operator correspondence relations. We consider how the number-conserving normally ordered products of the creation and annihilation operators act on the nondiagonal 
Hartree-Fock projections (1):

$$
\begin{gathered}
\widehat{a}_{k_{1}}^{\dagger} \ldots \widehat{a}_{k_{l}}^{\dagger} \widehat{a}_{k_{1}^{\prime}} \ldots \widehat{a}_{k_{l}^{\prime}} \widehat{\Lambda}\left(\boldsymbol{\phi}, \boldsymbol{\phi}^{+*}\right) \\
=\phi_{k_{1}^{\prime}} \ldots \phi_{k_{l}^{\prime}} \frac{\delta}{\delta \phi_{k_{1}}} \ldots \frac{\delta}{\delta \phi_{k_{l}}} \widehat{\Lambda}\left(\boldsymbol{\phi}, \boldsymbol{\phi}^{+*}\right), \\
\widehat{\Lambda}\left(\boldsymbol{\phi}, \boldsymbol{\phi}^{+*}\right) \widehat{a}_{k_{1}}^{\dagger} \ldots \widehat{a}_{k_{l}}^{\dagger} \widehat{a}_{k_{1}^{\prime}} \ldots \widehat{a}_{k_{l}^{\prime}} \\
=\phi_{k_{1}}^{+*} \ldots \phi_{k_{l}}^{+*} \frac{\delta}{\delta \phi_{k_{1}^{\prime}}^{+*}} \ldots \frac{\delta}{\delta \phi_{k_{l}^{\prime}}^{+*}} \widehat{\Lambda}\left(\boldsymbol{\phi}, \boldsymbol{\phi}^{+*}\right) .
\end{gathered}
$$

Here, the symbol

$$
\frac{\delta}{\delta \phi_{k}}=\frac{1}{\Delta V} \frac{\partial}{\partial \phi_{k}}
$$

denotes the lattice counterpart of the variational derivative. We substitute the relations (39) and (40) into the diagonal Hartree-Fock expansion (17) for $\widehat{\rho}(t)$. For the relation (39) we obtain the correspondence of the form

$$
\begin{aligned}
\widehat{a}_{k_{1}}^{\dagger} \ldots \widehat{a}_{k_{l}}^{\dagger} \widehat{a}_{k_{1}^{\prime}} \ldots \widehat{a}_{k_{l}^{\prime}} \widehat{\rho}(t)= & \int \prod_{k=1}^{\mathcal{N}} d \phi_{k} d \phi_{k}^{*} \mathcal{P}\left(\boldsymbol{\phi}, \boldsymbol{\phi}^{*}\right) \\
& \times \phi_{k_{1}^{\prime}} \ldots \phi_{k_{l}^{\prime}} \frac{\delta}{\delta \phi_{k_{1}}} \ldots \frac{\delta}{\delta \phi_{k_{l}}} \widehat{\Lambda}\left(\boldsymbol{\phi}, \boldsymbol{\phi}^{*}\right) .
\end{aligned}
$$

For the relation (40) we obtain the correspondence analogous to (42). Note that here we drop the condition $\|\boldsymbol{\phi}\|=1 / \sqrt{\Delta V}$ in (42). Now, suppose that $\mathcal{P}\left(\boldsymbol{\phi}, \boldsymbol{\phi}^{*}\right)$ is decreasing sufficiently rapidly as $\|\boldsymbol{\phi}\| \rightarrow \infty$, so that we can perform integration by parts, and the boundary terms vanish. Then, we obtain the mappings

$$
\begin{aligned}
\widehat{a}_{k_{1}}^{\dagger} \ldots \widehat{a}_{k_{l}}^{\dagger} \widehat{a}_{k_{1}^{\prime}} \ldots \widehat{a}_{k_{l}^{\prime}} \widehat{\rho}(t) \\
=\int \prod_{k=1}^{\mathcal{N}} d \phi_{k} d \phi_{k}^{*}|N: \boldsymbol{\phi}\rangle\langle N: \boldsymbol{\phi}| \\
\quad \times(-1)^{l} \frac{\delta}{\delta \phi_{k_{1}}} \ldots \frac{\delta}{\delta \phi_{k_{l}}} \phi_{k_{1}^{\prime}} \ldots \phi_{k_{l}^{\prime}} \mathcal{P}\left(\boldsymbol{\phi}, \boldsymbol{\phi}^{*}\right), \\
\widehat{\rho}(t) \widehat{a}_{k_{1}}^{\dagger} \ldots \widehat{a}_{k_{l}}^{\dagger} \widehat{a}_{k_{1}^{\prime}} \ldots \widehat{a}_{k_{l}^{\prime}} \\
=\int \prod_{k=1}^{\mathcal{N}} d \phi_{k} d \phi_{k}^{*}|N: \boldsymbol{\phi}\rangle\langle N: \boldsymbol{\phi}| \\
\quad \times(-1)^{l} \frac{\delta}{\delta \phi_{k_{1}^{\prime}}^{*}} \ldots \frac{\delta}{\delta \phi_{k_{l}^{\prime}}^{*}} \phi_{k_{1}}^{*} \ldots \phi_{k_{l}}^{*} \mathcal{P}\left(\boldsymbol{\phi}, \boldsymbol{\phi}^{*}\right) .
\end{aligned}
$$

If we have not dropped the condition $\|\boldsymbol{\phi}\|=1 / \sqrt{\Delta V}$, then the boundary terms would appear for sure. To ensure the absence of the boundary terms, it is sufficient to assume that $\mathcal{P}\left(\boldsymbol{\phi}, \boldsymbol{\phi}^{*}\right)$ and all of its derivatives are decreasing faster than any inverse power law. In mathematical words, we assume that $\mathcal{P}\left(\boldsymbol{\phi}, \boldsymbol{\phi}^{*}\right)$ belongs to the Schwartz space $\mathcal{S}\left[\mathbb{C}^{\mathcal{N}}\right]$ of all rapidly decreasing functions on $\mathbb{C}^{\mathcal{N}}$ [37]. Now, we substitute the correspondence relations (43) and (44) into the quantum
Liouville equation (38) for the Hamiltonian (37), and get

$$
\begin{aligned}
& \int \prod_{k=1}^{\mathcal{N}} d \phi_{k} d \phi_{k}^{*}|N: \boldsymbol{\phi}\rangle\langle N: \boldsymbol{\phi}| \\
& \quad \times\left\{\frac{\partial}{\partial t}+\Delta V^{2} \frac{1}{i \hbar} \sum_{r, s} h_{r s}\left[\frac{\delta}{\delta \phi_{r}} \phi_{s}-\frac{\delta}{\delta \phi_{s}^{*}} \phi_{r}^{*}\right]\right. \\
& \quad-\frac{1}{2} \Delta V^{2} \frac{1}{i \hbar} \sum_{r, s} V_{r s}\left[\frac{\delta}{\delta \phi_{r}} \frac{\delta}{\delta \phi_{s}} \phi_{s} \phi_{r}\right. \\
&\left.\left.-\frac{\delta}{\delta \phi_{r}^{*}} \frac{\delta}{\delta \phi_{s}^{*}} \phi_{s}^{*} \phi_{r}^{*}\right]\right\} \mathcal{P}\left(\boldsymbol{\phi}, \boldsymbol{\phi}^{*}\right)=0 .
\end{aligned}
$$

To make the notation more succinct, we introduce the vector

$$
\mathcal{A}_{r}[\phi]=\frac{1}{i \hbar} \Delta V \sum_{s} h_{r s} \phi_{s}
$$

and the matrix operator

$$
D_{r s}=D_{r s}[\phi]=\frac{\phi_{r}}{\sqrt{i \hbar}} V_{r s} \frac{\phi_{s}}{\sqrt{i \hbar}} .
$$

One of the solutions of the integral equation (45) is obtained by equating the coefficients of $|N: \boldsymbol{\phi}\rangle\langle N: \boldsymbol{\phi}|$ :

$$
\begin{aligned}
\frac{\partial}{\partial t} \mathcal{P}= & -\Delta V \sum_{r} \frac{\delta}{\delta \phi_{r}}\left\{\mathcal{A}_{r}[\boldsymbol{\phi}] \mathcal{P}\right\} \\
& -\Delta V \sum_{r} \frac{\delta}{\delta \phi_{r}^{*}}\left\{\left(\mathcal{A}_{r}[\boldsymbol{\phi}]\right)^{*} \mathcal{P}\right\} \\
& +\frac{1}{2} \Delta V^{2} \sum_{r, s} \frac{\delta}{\delta \phi_{r}} \frac{\delta}{\delta \phi_{s}}\left\{D_{r s} \mathcal{P}\right\} \\
& +\frac{1}{2} \Delta V^{2} \sum_{r, s} \frac{\delta}{\delta \phi_{r}^{*}} \frac{\delta}{\delta \phi_{s}^{*}}\left\{\left(D_{r s}\right)^{*} \mathcal{P}\right\} .
\end{aligned}
$$

This equation defines a certain time evolution for $\mathcal{P}$. Therefore, the validity of Eq. (48) is based on the assumption that the property of $\mathcal{P}$ belonging to the Schwartz space $\mathcal{S}\left[\mathbb{C}^{\mathcal{N}}\right]$ is invariant under this evolution. To bring our notation into better agreement with the emerging mathematical structure, we introduce the following definitions. For any complex vector $\boldsymbol{\theta}$ from $\mathbb{C}^{\mathcal{N}}$ we define the vector $\underline{\theta}=\left(\boldsymbol{\theta}, \boldsymbol{\theta}^{*}\right)^{T}$ with components $\underline{\theta}_{\alpha r}=\theta_{r}$ for $\alpha=1$ and $\underline{\theta}_{\alpha r}=\bar{\theta}_{r}^{*}$ for $\alpha=2$. Then, for the vector $\mathcal{A}_{r}[\phi]$ we have $\underline{\mathcal{A}}[\phi]=\left(\mathcal{A}_{r}[\phi],\left(\mathcal{A}_{r}[\phi]\right)^{*}\right)^{T}$. The matrices $\underline{\underline{D}}$ which act on such vectors have the components $\underline{\underline{\mathcal{D}}}_{\alpha \beta r s}$ :

$$
\underline{\underline{\mathcal{D}}}=\left[\begin{array}{cc}
P & Q \\
Q^{*} & P^{*}
\end{array}\right],
$$

where $P$ and $Q$ are arbitrary complex $\mathcal{N} \times \mathcal{N}$ matrices. Here, the greek indices $\alpha, \beta$ refer to the block submatrices, and the latin indices $r, s$ refer to the element in the selected block submatrix. Therefore, we treat the pairs $(\alpha, r)$ and $(\beta, s)$ as multi-indices. (Our definitions are inspired by the notation used in $[5,12]$.) Using these definitions, we rewrite 
the solution (48) as

$$
\begin{aligned}
\frac{\partial}{\partial t} \mathcal{P}= & -\Delta V \sum_{\alpha, r} \frac{\delta}{\delta \underline{\phi}_{\alpha r}}\left\{\underline{\mathcal{A}}_{\alpha r}[\underline{\phi}] \mathcal{P}\right\} \\
& +\frac{1}{2} \Delta V^{2} \sum_{\substack{\alpha, \beta \\
r, s}} \frac{\delta}{\delta \underline{\phi}_{\alpha r}} \frac{\delta}{\delta \underline{\phi}_{\beta s}^{*}}\left\{\underline{\underline{\mathcal{D}}}_{\alpha \beta r s}[\underline{\phi}] \mathcal{P}\right\},
\end{aligned}
$$

where the diffusion matrix $\underline{\mathcal{D}}$ is

$$
\underline{\underline{D}}=\left[\begin{array}{cc}
0 & D_{r s} \\
\left(D_{r s}\right)^{*} & 0
\end{array}\right] .
$$

Equation (50) has the form of the Fokker-Planck equation in terms of complex variables. We refer the reader to the Appendix B where the properties of such Fokker-Planck equations are summarized. We note that the drift term in (50) is in a correct form, and the matrix $\underline{\mathcal{D}}$ is Hermitian. However, for Eq. (50) to be a genuine Fokker-Planck equation in complex variables, the diffusion matrix $\mathcal{D}$ should be positive (semi)definite. The necessary condition $\overline{(\mathrm{B}} 21)$ for the positive semidefiniteness is violated since in Eq. (48) there are no terms like $\delta^{2} / \delta \phi_{r} \delta \phi_{s}^{*}$. Hence, Eq. (50) generally does not admit stochastic interpretation.

Despite the fact that the master equation of the diagonal representation (17) cannot be used for stochastic simulations of quantum dynamics, the representation (17) is useful when representing the initial conditions for the density operator $\hat{\rho}$.

\section{NONDIAGONAL HARTREE-FOCK STATE PROJECTION REPRESENTATION}

The nondiagonal coherent state projection representation, also called the positive $P$ representation [2,12], has been devised in order to solve the problem of the stochastical interpretability of the diagonal coherent state projection representation, or $P$ representation [1]. In our case for the diagonal Hartree-Fock state projection representation the same procedure has been performed by Carusotto et al. $[13,28]$ : the nondiagonal Hartree-Fock state projection representation is introduced:

$$
\begin{aligned}
P_{N} \hat{\rho} P_{N}= & \int \prod_{k=1}^{\mathcal{N}} d \phi_{k} d \phi_{k}^{*} \prod_{k=1}^{\mathcal{N}} d \phi_{k}^{+} d \phi_{k}^{+*} \\
& \times \mathcal{P}\left(\boldsymbol{\phi}, \boldsymbol{\phi}^{*}, \boldsymbol{\phi}^{+}, \boldsymbol{\phi}^{+*}\right)|N: \boldsymbol{\phi}\rangle\left\langle N: \boldsymbol{\phi}^{+}\right|
\end{aligned}
$$

Such a representation always exists for any physical numberconserving density operator in the $N$-particle subspace since we can always choose $\mathcal{P}$ as

$$
\begin{aligned}
& \mathcal{P}\left(\boldsymbol{\phi}, \boldsymbol{\phi}^{*}, \boldsymbol{\phi}^{+}, \boldsymbol{\phi}^{+*}\right) \\
& \quad=\delta_{S}\left(\|\boldsymbol{\phi}\|-\frac{1}{\Delta V}\right) \delta\left(\boldsymbol{\phi}^{+}-\boldsymbol{\phi}\right) \mathcal{P}_{\mathrm{diag}}\left(\boldsymbol{\phi}, \boldsymbol{\phi}^{*}\right),
\end{aligned}
$$

where $\mathcal{P}_{\text {diag }}\left(\phi, \phi^{*}\right)$ is the quasiprobability for the diagonal Hartree-Fock state representation (17). Here, $\delta_{S}$ is the surface delta function in $\mathbb{R}^{2 \mathcal{N}}$, and

$$
\delta\left(\boldsymbol{\phi}^{+}-\boldsymbol{\phi}\right)=\prod_{k=1}^{\mathcal{N}} \frac{1}{2} \delta\left(\phi_{k}^{+x}-\phi_{k}^{x}\right) \delta\left(\phi_{k}^{+y}-\phi_{k}^{y}\right) .
$$

Hereinafter, we employ the more compact notation $\mathcal{P}\left(\phi, \phi^{+}\right)=\mathcal{P}\left(\boldsymbol{\phi}, \boldsymbol{\phi}^{*}, \boldsymbol{\phi}^{+}, \boldsymbol{\phi}^{+*}\right)$.

The nondiagonal representation (52) can always be chosen positive. To show this, we use the trick of Carusotto et al. [13,28]. We start from the representation (52) and separate the phase of $\mathcal{P}$ :

$$
\mathcal{P}\left(\underline{\phi}, \underline{\phi}^{+}\right)=\left|\mathcal{P}\left(\underline{\phi}, \underline{\phi}^{+}\right)\right| e^{i \xi\left(\underline{\phi}, \underline{\phi}^{+}\right)} .
$$

Now, we note that we can always absorb the phase into $|N: \boldsymbol{\phi}\rangle\left\langle N: \boldsymbol{\phi}^{+}\right|$:

$$
\begin{aligned}
P_{N} \hat{\rho} P_{N}= & \int \prod_{k=1}^{\mathcal{N}} d \phi_{k} d \phi_{k}^{*} \prod_{k=1}^{\mathcal{N}} d \phi_{k}^{+} d \phi_{k}^{+*}\left|\mathcal{P}\left(\underline{\phi}, \underline{\phi}^{+}\right)\right| \\
& \times\left|N: \phi e^{i \xi\left(\underline{\phi}, \underline{\phi}^{+}\right) / 2 N}\right\rangle\left\langle N: \phi^{+} e^{-i \xi\left(\underline{\phi}, \underline{\phi}^{+}\right) / 2 N}\right|
\end{aligned}
$$

If we change the variables as $\boldsymbol{\phi} e^{i \xi\left(\phi, \underline{\phi}^{+}\right) / 2 N} \rightarrow \boldsymbol{\phi}$, $\boldsymbol{\phi}^{+} e^{-i \xi\left(\underline{\phi}, \underline{\phi}^{+}\right) / 2 N} \rightarrow \boldsymbol{\phi}^{+}$, and compute the Jacobian determinant, we can return to the form (52) where the $\mathcal{P}$ is now real and positive. However, from the point of view of the stochastic simulations, one may stop at (56): we sample the initial conditions with the probability distribution $\left|\mathcal{P}\left(\phi, \phi^{+}\right)\right|$, and each time we multiply the sampled wave functions by the phase factor $e^{ \pm i \xi\left(\underline{\phi}, \underline{\phi}^{+}\right) / 2 N}$.

\section{A. Quasiprobability master equation}

\section{Operator mappings of the star-product type}

If we reiterate the steps (39)-(48) of the derivation of the Fokker-Planck equation (50) for the case of nondiagonal Hartree-Fock representation (52), under the same assumptions about the absence of the boundary terms, the following operator mappings are obtained:

$$
\begin{aligned}
\widehat{a}_{k_{1}}^{\dagger} \ldots \widehat{a}_{k_{l}}^{\dagger} \widehat{a}_{k_{1}^{\prime}} \ldots \widehat{a}_{k_{l}^{\prime}} \widehat{\rho}(t) \\
\longleftrightarrow(-1)^{l} \frac{\delta}{\delta \phi_{k_{1}}} \ldots \frac{\delta}{\delta \phi_{k_{l}}}\left\{\phi_{k_{1}^{\prime}} \ldots \phi_{k_{l}^{\prime}} \mathcal{P}\left(\underline{\phi}, \underline{\phi}^{+}\right)\right\} \\
\widehat{\rho}(t) \widehat{a}_{k_{1}}^{\dagger} \ldots \widehat{a}_{k_{l}}^{\dagger} \widehat{a}_{k_{1}^{\prime}} \ldots \widehat{a}_{k_{l}^{\prime}} \\
\longleftrightarrow(-1)^{l} \frac{\delta}{\delta \phi_{k_{1}^{\prime}}^{+*}} \ldots \frac{\delta}{\delta \phi_{k_{l}^{\prime}}^{+*}}\left\{\phi_{k_{1}}^{+*} \ldots \phi_{k_{l}}^{+*} \mathcal{P}\left(\underline{\phi}, \underline{\phi}^{+}\right)\right\}
\end{aligned}
$$

Now, consider an arbitrary number-conserving observable $O\left(\widehat{\mathbf{a}}^{\dagger}, \widehat{\mathbf{a}}\right)$, defined as normally ordered power-series expansion in the components $\widehat{a}_{k}$ and $\widehat{a}_{k}^{\dagger}$ of the vectors $\widehat{\mathbf{a}}=\left(\widehat{a}_{1}, \ldots, \widehat{a}_{\mathcal{N}}\right)$ and $\widehat{\mathbf{a}}^{\dagger}$. Then, we have the mappings for $O\left(\widehat{\mathbf{a}}^{\dagger}, \widehat{\mathbf{a}}\right)$ :

$$
\begin{array}{r}
O\left(\widehat{\mathbf{a}}^{\dagger}, \widehat{\mathbf{a}}\right) \widehat{\rho}(t) \longleftrightarrow O\left(-\frac{\delta}{\delta \boldsymbol{\phi}}, \boldsymbol{\phi}\right) \mathcal{P}\left(\underline{\phi}, \underline{\phi}^{+}\right), \\
\widehat{\rho}(t) O\left(\widehat{\mathbf{a}}^{\dagger}, \widehat{\mathbf{a}}\right) \longleftrightarrow \mathcal{P}\left(\underline{\phi}, \underline{\phi}^{+}\right) O\left(\boldsymbol{\phi}^{+*},-\frac{\overleftarrow{\delta}}{\delta \boldsymbol{\phi}^{+*}}\right) .
\end{array}
$$

Note that the last two relations (59) and (60) bear strong resemblance to the star product in the deformation quantization [38]. In fact, we believe that this resemblance can be elaborated further, and therefore we call the relations (57) and (59) the "left star-product operator mappings," and the relations (58) and (60) the "right star-product operator mappings" correspondingly. 


\section{Master equation}

Employing the star-product operator mappings (57) and (58) in the quantum Liouville equation (38), we obtain the master equation

$$
\begin{aligned}
\frac{\partial}{\partial t} \mathcal{P}= & -\Delta V \sum_{r} \frac{\delta}{\delta \phi_{r}}\left\{\mathcal{A}_{r}[\boldsymbol{\phi}] \mathcal{P}\right\}-\Delta V \sum_{r} \frac{\delta}{\delta \phi_{r}^{+*}}\left\{\left(\mathcal{A}_{r}\left[\boldsymbol{\phi}^{+}\right]\right)^{*} \mathcal{P}\right\}+\frac{1}{2} \Delta V^{2} \sum_{r, s} \frac{\delta}{\delta \phi_{r}} \frac{\delta}{\delta \phi_{s}}\left\{V_{r s} \frac{\phi_{r} \phi_{s}}{i \hbar} \mathcal{P}\right\} \\
& +\frac{1}{2} \Delta V^{2} \sum_{r, s} \frac{\delta}{\delta \phi_{r}^{+*}} \frac{\delta}{\delta \phi_{s}^{+*}}\left\{\left(V_{r s} \frac{\phi_{r}^{+} \phi_{s}^{+}}{i \hbar}\right)^{*} \mathcal{P}\right\} .
\end{aligned}
$$

According to Appendix B, this equation differs from a genuine Fokker-Planck form since it lacks the complex conjugates of the drift terms at the right-hand side. Moreover, the diffusion matrix for $\phi$,

$$
\underline{\underline{D}}=\left[\begin{array}{cc}
0 & V_{r s} \frac{\phi_{r} \phi_{s}}{i \hbar} \\
0 & 0
\end{array}\right]
$$

and the diffusion matrix for $\boldsymbol{\phi}^{+}$,

$$
\underline{\underline{\mathcal{D}}}^{+}=\left[\begin{array}{cc}
0 & 0 \\
\left(V_{r s} \frac{\phi_{r}^{+} \phi_{s}^{+}}{i \hbar}\right)^{*} & 0
\end{array}\right]
$$

are not even Hermitian. In the following subsections, we will show how the nonuniqueness of the nondiagonal representation (52) can be used in order to transform the equation (61) into the genuine Fokker-Planck form.

\section{B. Linear automorphisms of the nondiagonal Hartree-Fock state projection representation}

In principle, in order to characterize the nonuniqueness of the nondiagonal representation (52), we can find the relevant spaces $R$ and $Z$ by applying the methods analogous to those used in Sec. III B. But, in fact it is not sufficient for our needs. Indeed, when we add some element $\mathcal{Z}$ of the space $Z$ to the quasiprobability functional $\mathcal{P}$, we actually add some inhomogeneous term to the master equation (61). However, in order to fix the drift and diffusion terms in (61), we need the possibility of addition to the Eq. (61) of the terms of the multiplicative form $\lambda^{T} P$, where $\lambda^{T}: D \rightarrow D$ is some linear operator acting on the space $D$ of all the quasiprobability functionals $\mathcal{P}\left(\phi, \phi^{+}\right)$. Therefore, we need to characterize the semigroup of linear automorphisms of the quasiprobability representation (52).

Annihilators of overcomplete basis. Let us write the nondiagonal representation (52) as the inner product

$$
P_{N} \hat{\rho} P_{N}=\left\langle\mathcal{P}^{*} \mid \widehat{\Lambda}\right\rangle
$$

For each overcomplete basis we have the decomposition (26). Therefore, we can introduce a space $A$ of all the linear operators such that for each $\lambda \in A$ we have (i) $R \subset \operatorname{ker} \lambda^{*}$ and (ii) $\lambda$ does not produce the boundary terms in the course of partial integration for all sufficiently rapidly decreasing functionals $\mathcal{P}$ (again, to be specific, we can choose the space $D$ as $\left.D=\mathcal{S}\left[\mathbb{C}^{\mathcal{N}}\right]\right)$. Then, we can consider the adjoint operators $\lambda^{\dagger}:\left\langle\mathcal{P}^{*} \mid \lambda \widehat{\Lambda}\right\rangle=\left\langle\lambda^{\dagger} \mathcal{P}^{*} \mid \widehat{\Lambda}\right\rangle$. It can be seen that $A$ is the space of all such linear operators $\lambda$ that

$$
\lambda \widehat{\Lambda}=0,
$$

where $\widehat{\Lambda}$ is treated as a functional of $\left(\phi, \phi^{+}\right)$, and $\lambda$ adhere to the same condition (ii) on the absence of boundary terms. According to the conventions adopted in mathematics, we call $A$ the space of annihilators of a given overcomplete basis. Practically, this means that for a given overcomplete basis we always have certain integrodifferential identities of the form (65). In our case of the Hartree-Fock state projections (1), these identities are the analyticity of the basis $\widehat{\Lambda}\left(\boldsymbol{\phi}, \boldsymbol{\phi}^{+*}\right)$ with respect to its variables

$$
\frac{\delta}{\delta \phi_{k}^{*}} \widehat{\Lambda}\left(\boldsymbol{\phi}, \boldsymbol{\phi}^{+*}\right)=0, \quad \frac{\delta}{\delta \phi_{k}^{+}} \widehat{\Lambda}\left(\boldsymbol{\phi}, \boldsymbol{\phi}^{+*}\right)=0 .
$$

Another important differential identity is

$$
\begin{gathered}
{\left[N-\Delta V \sum_{k} \phi_{k} \frac{\delta}{\delta \phi_{k}}\right] \hat{\Lambda}\left(\boldsymbol{\phi}, \boldsymbol{\phi}^{+*}\right)=0,} \\
{\left[N-\Delta V \sum_{k} \phi_{k}^{+*} \frac{\delta}{\delta \phi_{k}^{+*}}\right] \hat{\Lambda}\left(\boldsymbol{\phi}, \boldsymbol{\phi}^{+*}\right)=0,}
\end{gathered}
$$

which stems from the homogeneity of the Hartree-Fock state. We call this property the "absorption property" due to the reasons which will become evident in Sec. IV D.

Now consider the space $A^{T}$ of all the operators $\lambda^{T}$, where $\lambda \in A$. It can be shown (by partial integration) that $A^{T}$ is the space of all such operators $\lambda^{T}$ that their images are included in $Z, \operatorname{im} \lambda^{T} \subset Z$. Therefore, for any quasiprobability functional $\mathcal{P}\left(\underline{\phi}, \underline{\phi}^{+}\right)$and for any $\lambda^{T} \in A^{T}$ we have $\lambda^{T} \mathcal{P} \in Z$, i.e., $\lambda^{T} \mathcal{P}$ corresponds to zero operator in the nondiagonal expansion (52). Practically, this means that if we know the annihilators $\lambda$ of a given overcomplete basis, we can add the terms $\lambda^{T} \mathcal{P}$ to the right-hand side of the master equation (61), in order to modify the drift and diffusion terms, and hence to put this equation into the form of a genuine Fokker-Planck equation, according to the Appendix B. In our case of the basis $\widehat{\Lambda}\left(\boldsymbol{\phi}, \boldsymbol{\phi}^{+*}\right)$, due to the annihilators (66)-(68), the $\lambda^{T}$ assumes the form

$$
\begin{aligned}
\lambda^{T}= & \Delta V \sum_{k} \frac{\delta}{\delta \phi_{k}^{*}} l_{k}+\Delta V \sum_{k} \frac{\delta}{\delta \phi_{k}^{+}} l_{k}^{+}+N d \\
& +\Delta V \sum_{k} \frac{\delta}{\delta \phi_{k}} \phi_{k} d+N d^{+*}+\Delta V \sum_{k} \frac{\delta}{\delta \phi_{k}^{+*}} \phi_{k}^{+*} d^{+*}
\end{aligned}
$$


where $l_{k}, l_{k}^{+}, d, d^{+}$are some linear operators $D \rightarrow D$ which do not produce the boundary terms in the course of partial integration. We will employ $\lambda^{T}$ in Eq. (61) in the following section.

Gauge theory structure. In the remainder of this section, we go a little bit further and develop geometrical interpretation of the nonuniqueness offered by the space of annihilators $A$. Note that $A^{T}$ possesses the structure of semigroup: if $\lambda_{1}^{T} \in A^{T}$ and $\lambda_{2}^{T} \in A^{T}$, then $\lambda_{1}^{T} \lambda_{2}^{T} \in A^{T}$. Consider the semigroup $G$ generated by all the operators

$$
g=1+\lambda^{T}
$$

where $\lambda^{T} \in A^{T}$. Then, $G$ is a semigroup of linear automorphisms of the quasiprobability representations, i.e., for any $g \in G$ and for any $\mathcal{P}$, the functionals $\mathcal{P}$ and $g \mathcal{P}$ belong to the same equivalence class. This means that when we map the quantum Liouville equation (38) into the phase-space master equation (61), we can choose the arbitrary automorphism $g$ for each additive term individually. The automorphism $g$ can even be varied with time, $g=g(t)$. Therefore, our phasespace representation has the structure of gauge theory [12]. According to the conventions adopted in physical gauge theories, $G$ is called the gauge symmetry group; $g \in G$ is called the gauge transformation; $\lambda^{T}$ is called the gauge; $l_{k}, l_{k}^{+}$, $d, d^{+}$in Eq. (69) are called the gauge parameters.

\section{Stochastic unraveling of the master equation}

Now, we turn to the master equation (61). The form (69) of the gauge $\lambda^{T}$ suggests that we can add such terms to the equation (61) that the correct form of the drift terms and the correct Hermitian symmetry of the diffusion matrices are restored. After having performed that, the master equation (61) becomes

$$
\begin{aligned}
\frac{\partial}{\partial t} \mathcal{P}= & -\Delta V \sum_{\alpha r} \frac{\delta}{\delta \underline{\phi}_{\alpha r}}\left\{\underline{\mathcal{A}}_{\alpha r}[\underline{\phi}] \mathcal{P}\right\}+\frac{1}{2} \Delta V^{2} \sum_{\alpha, \beta} \frac{\delta}{\delta \underline{\phi}_{\alpha r}} \frac{\delta}{\delta \underline{\phi}_{\beta s}^{*}}\left\{\underline{\underline{D}}_{\alpha \beta r s}[\underline{\phi}] \mathcal{P}\right\}-\Delta V \sum_{\alpha r} \frac{\delta}{\delta \underline{\phi}_{\alpha r}^{+}}\left\{\underline{\mathcal{A}}_{\alpha r}\left[\underline{\phi}^{+}\right] \mathcal{P}\right\} \\
& +\frac{1}{2} \Delta V^{2} \sum_{\substack{\alpha, \beta \\
r, s}} \frac{\delta}{\delta \underline{\phi}_{\alpha r}^{+}} \frac{\delta}{\delta \underline{\phi}_{\beta s}^{+*}}\left\{\underline{\underline{\mathcal{D}}}_{\alpha \beta r s}\left[\underline{\phi}^{+}\right] \mathcal{P}\right\}
\end{aligned}
$$

where the diffusion matrices now have the form

$$
\underline{\underline{D}} \underline{\phi}]=\left[\begin{array}{cc}
\frac{\phi_{r}}{\sqrt{i \hbar}} V_{r s}^{(1)} \frac{\phi_{s}^{*}}{\sqrt{-i \hbar}} & \frac{\phi_{r}}{\sqrt{i \hbar}} V_{r s} \frac{\phi_{s}}{\sqrt{i \hbar}} \\
\frac{\phi_{r}^{*}}{\sqrt{-i \hbar}} V_{r s}^{*} \frac{\phi_{s}^{*}}{\sqrt{-i \hbar}} & \frac{\phi_{r}^{*}}{\sqrt{-i \hbar}} V_{r s}^{(1) * \frac{\phi_{s}}{\sqrt{i \hbar}}}
\end{array}\right] .
$$

We see that $\underline{\underline{D}}[\phi]$ is Hermitian if the matrix $V_{r s}^{(1)}$ is Hermitian. The matrix $V_{r s}^{(1)}$ remains to be determined to satisfy the condition of positive (semi)definiteness. It turns out that it is easier to factorize the diffusion matrix $\underline{\mathcal{D}}$ with the Ito process [35] driven by complex noise. Such stochastic processes and the general forms of their diffusion matrices are reviewed in Appendix B 2. From the main result (B16) it follows that in order to factorize $\underline{\mathcal{D}}$, it is enough to represent the matrix $V_{r s}$ as $V=Y_{+} Y_{-}^{T}+\left(Y_{+} Y_{-}^{T}\right)^{T}$. Since $V_{r s}$ is a convolution operator, we have

$$
\begin{aligned}
V & =\sqrt{\mathcal{N}}\left(F^{-1}\right)_{p \rightarrow x} \widetilde{V}_{\mathrm{int}}(\boldsymbol{p}) F_{\boldsymbol{x} \rightarrow \boldsymbol{p}} \\
& =\sqrt{\mathcal{N}}\left(F^{-1}\right)_{\boldsymbol{p} \rightarrow \boldsymbol{x}} \widetilde{V}_{\mathrm{int}}^{1 / 2}(\boldsymbol{p}) \widetilde{V}_{\mathrm{int}}^{1 / 2}(\boldsymbol{p}) F_{\boldsymbol{x} \rightarrow \boldsymbol{p}}
\end{aligned}
$$

where the unitary discrete Fourier transform operator $F$ has the matrix elements

$$
\left\langle\boldsymbol{y}_{r}\left|F_{\boldsymbol{x} \rightarrow \boldsymbol{y}}\right| \boldsymbol{x}_{s}\right\rangle=\frac{1}{\sqrt{\mathcal{N}}} \exp \left[-i\left\langle\boldsymbol{x}_{s} \mid \boldsymbol{y}_{r}\right\rangle\right]
$$

The Fourier transform of the pair potential is $\widetilde{V}_{\text {int }}(\boldsymbol{p})=$ $F_{\boldsymbol{x} \rightarrow \boldsymbol{p}} V_{\text {int }}(\boldsymbol{x})$. From the factorization of $V(73)$ it is seen that

we can take

$$
Y_{+}=\mathcal{N}^{1 / 4}\left(F^{\dagger}\right)_{p \rightarrow x} \widetilde{V}_{\text {int }}^{1 / 2}(p) / \sqrt{2}
$$

and

$$
Y_{-}=\mathcal{N}^{1 / 4} F_{p \rightarrow x} \widetilde{V}_{\text {int }}^{1 / 2}(\boldsymbol{p}) / \sqrt{2} .
$$

As far as in this work we construct the stochastic interpretation to prove the concept, we do not investigate the efficiency (see Appendix B 3) of the factorization given by (75) and (76). Although we believe that such a factorization is close to optimal (and much better than the trivial factorization of Appendix B 3) as long as in the work of Carusotto et al. [13] it is shown that in the case of the pair potential with real positive $\widetilde{V}_{\text {int }}(\boldsymbol{p})$, the choice analogous to (75) and (76) is optimal. This choice corresponds to $V^{(1)}=Y_{+} Y_{+}^{\dagger}+Y_{-} Y_{-}^{\dagger}$, and the diffusion matrix $\underline{\underline{D}}$ is factorized with the noise matrix

$$
\underline{\underline{\mathcal{B}}} \underline{\underline{\phi}}]=\left[\begin{array}{cc}
\frac{\phi_{r}}{\sqrt{i \hbar}}\left(Y_{+}\right)_{r s} & \frac{\phi_{r}}{\sqrt{i \hbar}}\left(Y_{-}\right)_{r s} \\
\frac{\phi_{r}^{*}}{\sqrt{-i \hbar}}\left(Y_{-}^{*}\right)_{r s} & \frac{\phi_{r}^{*}}{\sqrt{-i \hbar}}\left(Y_{+}^{*}\right)_{r s}
\end{array}\right] .
$$

According to Appendix B, now Eq. (71) has the form of the genuine Fokker-Planck equation, where $\mathcal{P}$ is the joint probability distribution of the two independent stochastic processes $\phi$ and $\boldsymbol{\phi}^{+}$:

$$
\begin{aligned}
d \phi_{r}= & \mathcal{A}_{r}[\phi] d t+\frac{\phi_{r}}{\sqrt{i \hbar}} \sum_{s}\left(Y_{+}\right)_{r s} d Z_{s} \\
& +\frac{\phi_{r}}{\sqrt{i \hbar}} \sum_{s}\left(Y_{-}\right)_{r s} d Z_{s}^{*},
\end{aligned}
$$




$$
\begin{aligned}
d \phi_{r}^{+}= & \mathcal{A}_{r}\left[\phi^{+}\right] d t+\frac{\phi_{r}^{+}}{\sqrt{i \hbar}} \sum_{s}\left(Y_{+}\right)_{r s} d Z_{s}^{+} \\
& +\frac{\phi_{r}^{+}}{\sqrt{i \hbar}} \sum_{s}\left(Y_{-}\right)_{r s} d Z_{s}^{+*} .
\end{aligned}
$$

Here, the vector Wiener increments $d Z_{s}$ and $d Z_{s}^{+}$are complex valued, independent $\mathrm{E}\left[d \underline{Z}_{\alpha s} d \underline{Z}_{\beta r}^{+}\right]=0, \delta$ correlated $\mathrm{E}\left[d \underline{Z}_{\alpha s} d \underline{Z}_{\beta r}^{*}\right]=\mathrm{E}\left[d \underline{Z}_{\alpha s}^{+} d \underline{Z}_{\beta r}^{+*}\right]=\delta_{\alpha \beta} \delta_{s r} d t$, and have zero mean $\mathrm{E}\left[d Z_{s}\right]=\mathrm{E}\left[d Z_{s}^{+}\right]=0$.

\section{Optimization of the quasiprobability master equation}

Until now, we have used the gauge transformations generated by the analyticity property (66) to obtain the genuine stochastic representation of the quantum dynamics. According to the literature $[2,6,7,12]$, this analyticity is not specific to the Hartree-Fock state projections, but it is common to all the generalized phase-space methods which admit the diffusive stochastic representations. However, the Hartree-Fock state projections also have a specific differential property, namely, the "absorption property" (67) and (68). Let us investigate how we can benefit from the additional flexibility in the Fokker-Planck equation offered by the corresponding gauge terms in $\lambda^{T}$ (69). We will consider the two cases.

\section{Scalar gauge functions}

The first case is when the gauge parameter $d$ in Eq. (69) is a scalar function

$$
d=\omega\left(\underline{\phi}, \underline{\phi}^{+}\right)
$$

Since the variables $\phi$ and $\phi^{+}$enter the master equation in a symmetrical way, following we consider only the terms containing $\phi$. The results for $\phi^{+}$are obtained by the substitution $\phi \rightarrow \phi^{+}$. Under the gauge function $d$ [Eq. (80)] we can add to the Fokker-Planck equation the following terms:

$$
\begin{aligned}
& 0 \longleftrightarrow N \omega\left(\underline{\phi}, \underline{\phi}^{+}\right) \mathcal{P}\left(\underline{\phi}, \underline{\phi}^{+}\right) \\
&-\Delta V \sum_{k} \frac{\delta}{\delta \phi_{k}}\left\{-\phi_{k} \omega\left(\underline{\phi}, \underline{\phi}^{+}\right) \mathcal{P}\left(\underline{\phi}, \underline{\phi}^{+}\right)\right\} .
\end{aligned}
$$

If we add these terms to the master equation (61), then an additional term with the potential $N \omega\left(\phi, \phi^{+}\right)$appears in the resulting Fokker-Planck equation. According to the FeynmanKac formula [39], this means that our stochastic process becomes weighted. That is, we consider the stochastic process $\phi, \phi^{+}$for the Fokker-Planck equation with the potential term being dropped (but with all the other terms being kept). Then, the weight $\Omega(t)$ which is acquired along a particular random realization of the stochastic trajectory $\phi(t), \phi^{+}(t)$ is

$$
\Omega(t)=\exp \left[N \int_{0}^{t} \omega\left(\underline{\phi}(\tau), \underline{\phi}^{+}(\tau)\right) d \tau\right],
$$

and the quasiprobability distribution $\mathcal{P}\left(\phi^{\prime}, \phi^{+\prime}, t^{\prime}\right)$ (for the full Fokker-Planck equation with the potential term) at the phasespace point $\underline{\phi}^{\prime}, \underline{\phi}^{+\prime}$ and the time moment $t^{\prime}$ is given by the conditional expected value

$$
\mathcal{P}\left(\underline{\phi}^{\prime}, \underline{\phi}^{+\prime}, t^{\prime}\right)=\mathrm{E}\left[\Omega\left(t^{\prime}\right) \mid \underline{\phi}\left(t^{\prime}\right)=\underline{\phi}^{\prime}, \underline{\phi}^{+}\left(t^{\prime}\right)=\underline{\phi}^{+\prime}\right] .
$$

In summary, the scalar gauge transformation (81) means that we can absorb the part of the drift vector

$$
\mathcal{A}_{r}^{\Delta}\left[\underline{\phi}, \underline{\phi}^{+}\right]=\phi_{r} \omega\left(\underline{\phi}, \underline{\phi}^{+}\right)
$$

into the potential term

$$
\mathcal{V}^{\Delta}\left[\underline{\phi}, \underline{\phi}^{+}\right]=N \omega\left(\underline{\phi}, \underline{\phi}^{+}\right) .
$$

This flexibility may be useful when optimizing the Monte Carlo simulations of the stochastic differential equations (78) and (79), e.g., when it is necessary to remove or modify some parts of the drift vector.

\section{First-order differential gauge operators}

Now, we consider the case when the gauge parameter $d$ in (69) is a first-order differential operator

$$
d=-\Delta V \sum_{r} \frac{\delta}{\delta \phi_{r}} g_{r}\left(\underline{\phi}, \underline{\phi}^{+}\right) .
$$

For this choice of the gauge operator, the following terms can be added to the master equation (61):

$$
\begin{aligned}
0 \longleftrightarrow & -\Delta V \sum_{r} \frac{\delta}{\delta \phi_{r}}\left\{(N-1) g_{r} \mathcal{P}\left(\underline{\phi}, \underline{\phi}^{+}\right)\right\} \\
& +\frac{1}{2} \Delta V^{2} \sum_{r, s} \frac{\delta}{\delta \phi_{r}} \frac{\delta}{\delta \phi_{s}}\left\{-\left[\phi_{r} g_{s}+\phi_{s} g_{r}\right] \mathcal{P}\left(\underline{\phi}, \underline{\phi}^{+}\right)\right\},
\end{aligned}
$$

where compared to the general form (69) we have rearranged all the derivatives to the left using the commutation relations

$$
\phi_{k} \frac{\delta}{\delta \phi_{s}}=\frac{\delta}{\delta \phi_{s}} \phi_{k}-\frac{1}{\Delta V} \delta_{r, s},
$$

and have employed the symmetry of the mixed derivatives $\delta^{2} / \delta \phi_{r} \delta \phi_{s}$. This gauge transformation means that we can absorb the part of the diffusion matrix

$$
D_{r s}^{\Delta}=\left[\phi_{r} g_{s}+g_{r} \phi_{s}\right]
$$

into the drift vector

$$
\mathcal{A}_{r}^{\Delta}\left[\underline{\phi}, \underline{\phi}^{+}\right]=(N-1) g_{r}\left(\underline{\phi}, \underline{\phi}^{+}\right) .
$$

Factorization of the modified diffusion matrix. Once we apply the gauge transformation (89) and (90), we need to factorize the modified diffusion matrix

$$
D_{r s}^{\prime}=D_{r s}-\phi_{r} g_{s}-g_{r} \phi_{s},
$$

where $D_{r s}$ is the "bare" diffusion matrix (47). One particular case, when the factorization is evident, is when the gauge $g_{r}$ has the form

$$
|\boldsymbol{g}\rangle=B_{+}\left|\lambda_{-}\right\rangle+B_{-}\left|\lambda_{+}\right\rangle-|\phi\rangle\left\langle\lambda_{+}^{*} \mid \lambda_{-}\right\rangle,
$$

where $\lambda_{+}$and $\lambda_{-}$are arbitrary vectors (they may depend on $\phi$, $\phi^{+}$, and may vary with time); here, the bra-ket notation is for the lattice picture, as defined by Eq. (8); the noise matrices

$$
\left(B_{ \pm}\right)_{r s}=\frac{\phi_{r}}{\sqrt{i \hbar}}\left(Y_{ \pm}\right)_{r s}
$$


perform the factorization of the bare diffusion matrix $D_{r s}$ [see (77)]. Substituting the gauge parameter (92) into the expression (91), we find for $D_{r s}^{\prime}$

$$
D^{\prime}=B_{+}^{\prime} B_{-}^{\prime T}+B_{-}^{\prime} B_{+}^{\prime T},
$$

where the modified noise matrices

$$
B_{+}^{\prime}=B_{+}-|\phi\rangle\left\langle\lambda_{+}^{*}\left|, \quad B_{-}^{\prime}=B_{-}-\right| \phi\right\rangle\left\langle\lambda_{-}^{*}\right|
$$

thus perform the factorization of $D_{r s}^{\prime}$. After computing the modified drift vector $\mathcal{A}_{r}^{\prime}$,

$$
\mathcal{A}_{r}^{\prime}=\mathcal{A}_{r}+\mathcal{A}_{r}^{\Delta}=\mathcal{A}_{r}+(N-1) g_{r},
$$

we obtain the modified stochastic equation

$$
\begin{aligned}
d|\boldsymbol{\phi}\rangle= & \frac{1}{i \hbar} h|\boldsymbol{\phi}\rangle d t+(N-1)\left(B_{+}\left|\lambda_{-}\right\rangle+B_{-}\left|\lambda_{-}\right\rangle\right) d t \\
& -|\boldsymbol{\phi}\rangle(N-1)\left\langle\lambda_{+}^{*} \mid \lambda_{-}\right\rangle d t+\left(B_{+}-|\boldsymbol{\phi}\rangle\left\langle\lambda_{+}^{*}\right|\right) d \boldsymbol{Z} \\
& +\left(B_{-}-|\boldsymbol{\phi}\rangle\left\langle\lambda_{-}^{*}\right|\right) d \boldsymbol{Z}^{*} .
\end{aligned}
$$

The complex vector Wiener increments $d \boldsymbol{Z}$ and $d \boldsymbol{Z}^{+}$are now normalized as $\mathrm{E}\left[d \underline{Z}_{\alpha s} d \underline{Z}_{\beta r}^{*}\right]=\delta_{\alpha \beta} \delta_{s r} d t / \Delta V$. The stochastic equation for the $\phi^{+}$variable is obtained from (97) by attaching the supersript "+" wherever appropriate. The gauge parameters $\lambda_{ \pm}$and $\lambda_{ \pm}^{+}$(for $\boldsymbol{\phi}$ and $\boldsymbol{\phi}^{+}$variables, correspondingly) are generally independent of each other. Note that the "phasedrift" term

$$
\mathcal{A}_{r}^{\text {(phase })}=-\phi_{r}(N-1)\left\langle\lambda_{+}^{*} \mid \lambda_{-}\right\rangle
$$

in Eq. (97) can be absorbed into the potential term

$$
\mathcal{V}=-N(N-1)\left\langle\lambda_{+}^{*} \mid \lambda_{-}\right\rangle
$$

using the scalar gauge transformation (84) and (85).

At present, all the known in literature $[13,20,36]$ stochastic methods for the basis $\hat{\Lambda}$ can be rederived using the gauge parameters of a more special form

$$
\left|\lambda_{ \pm}\right\rangle=\frac{B_{ \pm}^{T}\left|\boldsymbol{\kappa}^{*}\right\rangle}{\langle\boldsymbol{\kappa} \mid \boldsymbol{\phi}\rangle}
$$

or, equivalently, when the gauge parameter $g_{r}$ has the form

$$
|\boldsymbol{g}\rangle=\left\{I-\frac{1}{2} \frac{|\boldsymbol{\phi}\rangle\langle\boldsymbol{\kappa}|}{\langle\boldsymbol{\kappa} \mid \boldsymbol{\phi}\rangle}\right\} \frac{D\left|\boldsymbol{\kappa}^{*}\right\rangle}{\langle\boldsymbol{\kappa} \mid \boldsymbol{\phi}\rangle},
$$

where $\boldsymbol{\kappa}$ is an arbitrary vector; $I$ is the identity operator in one-body Hilbert space $H_{\mathcal{N}}$. Then, introducing the projection

$$
Q_{\phi, \kappa}=I-\frac{|\boldsymbol{\phi}\rangle\langle\boldsymbol{\kappa}|}{\langle\boldsymbol{\kappa} \mid \boldsymbol{\phi}\rangle},
$$

we have for $D_{r s}^{\prime}$ in this case

$$
D^{\prime}=Q_{\phi, \kappa} D Q_{\phi, \kappa}^{T},
$$

and the modified noise matrices (95) assume the form

$$
B_{+}^{\prime}=Q_{\phi, \kappa} B_{+}, B_{-}^{\prime}=Q_{\phi, \kappa} B_{-} .
$$

The modified stochastic equation (97) now looks like

$$
\begin{aligned}
d|\boldsymbol{\phi}\rangle= & \frac{1}{i \hbar} h|\boldsymbol{\phi}\rangle d t+(N-1)\left\{I-\frac{1}{2} \frac{|\boldsymbol{\phi}\rangle\langle\boldsymbol{\kappa}|}{\langle\boldsymbol{\kappa} \mid \boldsymbol{\phi}\rangle}\right\} \frac{D\left|\boldsymbol{\kappa}^{*}\right\rangle}{\langle\boldsymbol{\kappa} \mid \boldsymbol{\phi}\rangle} d t \\
& +Q_{\phi, \kappa} B_{+} d \boldsymbol{Z}+Q_{\phi, \kappa} B_{-} d \mathbf{Z}^{*}
\end{aligned}
$$

The gauge transformations with $g_{r}$ of the form (101) have the semigroup property: if we apply any two such transformations consecutively, first the transformation $G_{\kappa_{1}}$ with the gauge parameter $\kappa_{1}$, and then the transformation $G_{\kappa_{2}}$ with the gauge parameter $\kappa_{2}$ (to the equation with the modified diffusion matrix after the first transformation), then the form of Eqs. (105) is retained, and it will have the parameter $\kappa=\kappa_{2}$ :

$$
G_{\kappa_{2}} \circ G_{\kappa_{1}}=G_{\kappa_{2}},
$$

which can be shown by direct algebraic calculation.

It is worth noting that the stochastic equations (105) are in fact the $\phi$ and $\boldsymbol{\phi}^{+}$parts of the stochastic Schrödinger equations (SSE) [20,25] for the single-body density matrices $\hat{\rho}_{1}=|\boldsymbol{\phi}\rangle\langle\boldsymbol{\kappa}|$ and $\hat{\rho}_{2}=\left|\boldsymbol{\phi}^{+}\right\rangle\left\langle\boldsymbol{\kappa}^{+}\right|$correspondingly. What is interesting in this result is that if we take the $\phi$ part from the SSE for $\hat{\rho}_{1}$, combine it with the $\boldsymbol{\phi}^{+}$part from the SSE for $\hat{\rho}_{2}$, then the resulting system of stochastic equations is an exact representation of the quantum dynamics for $\hat{\rho}=|\boldsymbol{\phi}\rangle\left\langle\boldsymbol{\phi}^{+}\right|$, with the gauge parameters $\left(\boldsymbol{\kappa}, \boldsymbol{\kappa}^{+}\right)$, which can be varied at will.

Here, it must be emphasized that the form (97) is only a special case of the general gauge $g_{r}$. Moreover, here we apply the gauges only to the bare diffusion matrices $D$ and $D^{+}$ separately. But, we can apply the gauges to the full diffusion matrix of the bare master equation

$$
\left[\begin{array}{cc}
D & 0 \\
0 & D^{+*}
\end{array}\right]
$$

for the variables $\boldsymbol{\phi}$ and $\boldsymbol{\phi}^{+*}$ (and thus mix these variables). Therefore, currently we cannot be sure that there is no other useful efficient gauge parameters that do not conform to the form (92). An additional investigation is required to study this more general case.

Now, we provide a few examples of how one can use the freedom offered by the transformations (89) and (90) to optimize the Monte Carlo simulation of the stochastic differential equations (78) and (79) for various purposes.

The simple scheme with Fock states of Carusotto et al. [13]. According to Appendix B 3, the efficiency of the simulation depends on the local growth rate $\sigma$ [Eq. (B18)] of the spread of the trajectories in the generalized phase space. Therefore, in order to make the simulation more efficient, we can minimize the diffusion term in the bare master equation (61). We find such a functional $g_{r}$ for which the matrix $D_{r s}^{\prime}$ [Eq. (91)] has the minimal Hilbert-Schmidt norm

$$
g_{r}=\arg \min \operatorname{Tr} D^{\prime} D^{\prime \dagger} .
$$

To find the vector $g_{r}$ satisfying (108), we solve the equation

$$
\frac{\delta}{\delta g_{r}} \operatorname{Tr} D^{\prime} D^{\prime \dagger}=0
$$

and find that the solution has the form (101) with $\kappa=\boldsymbol{\phi}$ and $\boldsymbol{\kappa}^{+}=\boldsymbol{\phi}^{+}$. If we substitute these values of $\boldsymbol{\kappa}$ and $\boldsymbol{\kappa}^{+}$into the modified stochastic equations (105), then the modified drift vector $\mathcal{A}_{r}^{\prime}$ becomes exactly the Gross-Pitaevskii term $F_{\alpha}^{\mathrm{GP}}$ from the work of Carusotto et al. [13], $\mathcal{A}_{r}^{\prime}=F_{\alpha}^{\mathrm{GP}}$, and the stochastic equations (105) reduce to the stochastic equations for the simple scheme with Fock states of Carusotto et al. [13], if the Fourier transform of the interaction potential $\widetilde{V}_{\text {int }}(\boldsymbol{p})$ is real. The numerical stability of this method is considered in Sec. V. 
The optimal observable evolution method of Lacroix [20]. As another example we consider the problem of constructing the trace-conserving stochastic representation. The conventional approach is to require the conservation of trace of the operator basis (1) along each random trajectory. That is, we require

$$
\operatorname{Tr} \hat{\Lambda}=N !\left\langle\boldsymbol{\phi}^{+} \mid \boldsymbol{\phi}\right\rangle^{N}=\mathrm{const},
$$

and hence we have the constraint

$$
f=\left\langle\boldsymbol{\phi}^{+} \mid \boldsymbol{\phi}\right\rangle=\text { const. }
$$

We compute the gradients $\delta f / \delta \phi=\left(\phi^{+*}, 0\right), \delta f / \delta \phi^{+}=$ $(0, \phi)$, and substitute them into the Ito's lemma (B17); from the stochastic part of the lemma we find the necessary conditions on the modified noise matrices:

$$
\begin{gathered}
\left\langle\boldsymbol{\phi}^{+}\right| B_{+}^{\prime}=0,\left\langle\boldsymbol{\phi}^{+}\right| B_{-}^{\prime}=0, \\
\langle\boldsymbol{\phi}| B_{+}^{+\prime}=0,\langle\boldsymbol{\phi}| B_{-}^{+\prime}=0 .
\end{gathered}
$$

From Eq. (104) we see that in order to satisfy these conditions, it is enough to set $\boldsymbol{\kappa}=\boldsymbol{\phi}^{+}$and $\boldsymbol{\kappa}^{+}=\boldsymbol{\phi}$. Then, in the deterministic part of the lemma (B17) the terms with second-order derivatives vanish, and the drift terms cancel each other for our choice of the gauge parameters $\left(\kappa, \kappa^{+}\right)$. The trace-conserving stochastic representation is constructed. If the phase-drift terms are converted into the potentials according to (98) and (99), they cancel each other, and the resulting stochastic equations coincide with that obtained in [20], except that the potential term $V_{r s}$ is factorized in a different way. The numerical stability of this method is considered in Sec. V.

The constant trace scheme with Fock states of Carusotto [13]. In the constant trace scheme of Lacroix [20], which was rederived in the preceding paragraph, the original (bare) diffusion matrix $D$ [Eq. (47)] is modified by the gauge transformation (89), (90), and (101) so as to make the noise orthogonal to the gradient of the trace. However, the same procedure can be applied not only to the bare matrix $D$ [Eq. (47)], but to any other (modified) diffusion matrix. For example, in the constant trace scheme with Fock states of Carusotto [13] this procedure is applied to the optimized diffusion matrix (103) of the simple scheme [13]. However, according to the semigroup property (106), the result is just the constant trace method of Lacroix [20], with the only minor difference being that the phase-drift terms (98) are not eliminated (which affects only the equal constant phase factors of $\phi$ and $\phi^{+}$). So, we conclude that the two methods, the optimal observable evolution method of Lacroix and the constant trace scheme with Fock states of Carusotto, are essentially the same for the operator basis (1). In this respect, it is interesting to note that the paper [20] argues that the two methods are different, due to the fact that it is not easy to grasp the structure and equivalence of the stochastic methods within the stochastic wave-function framework.

The numerical stability of this method is the same as that of the optimal observable evolution method of Lacroix.

\section{E. Evaluation of observable average values}

To evaluate the average value of the observable $\hat{O}$, we put it into the normal form $O\left(\widehat{\mathbf{a}}^{\dagger}, \widehat{\mathbf{a}}\right)$, and employ the trace formula

$$
\langle\hat{O}\rangle=\int \prod_{k=1}^{\mathcal{N}} d \phi_{k} d \phi_{k}^{*} \prod_{k=1}^{\mathcal{N}} d \phi_{k}^{+} d \phi_{k}^{+*} \mathcal{O}\left(\boldsymbol{\phi}, \boldsymbol{\phi}^{+*}\right) \mathcal{P}\left(\underline{\phi}, \underline{\phi}^{+}\right),
$$

where $\mathcal{O}$ is the phase-space representation of the observable

$$
\begin{aligned}
\mathcal{O}\left(\boldsymbol{\phi}, \boldsymbol{\phi}^{+*}\right) & =\operatorname{Tr}\left\{\widehat{\Lambda}\left(\phi, \phi^{+*}\right) O\left(\widehat{\mathbf{a}}^{\dagger}, \widehat{\mathbf{a}}\right)\right\} \\
& =O\left(\boldsymbol{\phi}^{+*}, \frac{\delta}{\delta \boldsymbol{\phi}^{+*}}\right) \operatorname{Tr}\left\{\widehat{\Lambda}\left(\phi, \phi^{+*}\right)\right\} .
\end{aligned}
$$

Here, in the last line in (114) we have used the operator mappings (40). In particular, since $\operatorname{Tr} \hat{\Lambda}=N !\left\langle\boldsymbol{\phi}^{+} \mid \boldsymbol{\phi}\right\rangle^{N}$, we have the operator mappings for the observable

$$
\begin{aligned}
& \widehat{a}_{k_{1}}^{\dagger} \ldots \widehat{a}_{k_{l}}^{\dagger} \widehat{a}_{k_{1}^{\prime}} \ldots \widehat{a}_{k_{m}^{\prime}} \\
& \quad \longleftrightarrow \delta_{l m} \phi_{k_{1}}^{+*} \ldots \phi_{k_{l}}^{+*} \phi_{k_{1}^{\prime}} \ldots \phi_{k_{m}^{\prime}} \frac{N !^{2}}{(N-m) !}\left\langle\boldsymbol{\phi}^{+} \mid \boldsymbol{\phi}\right\rangle^{N-m} .
\end{aligned}
$$

Although this result is known in the literature $[13,36]$, we deliberately derive it in a general way, so that it becomes evident how to proceed in the case of a general overcomplete operator basis, with well-defined operator correspondences: the operator mapping for observable is obtained by evaluating the action of the transposed star-product representation of the observable on the trace of the operator ansatz.

\section{PROBLEM OF BOUNDARY TERMS IN THE STOCHASTIC WAVE-FUNCTION METHODS}

Our rederivation of the stochastic wave-function method in the framework of generalized phase-space representation explicitly reveals that these methods are jeopardized by the problem of neglected boundary terms. In our rederivation, when we transform the star-product operator mappings for the basis $\hat{\Lambda}$ [Eqs. (39) and (40)] into the star-product mappings for the quasiprobability $\mathcal{P}$ [Eqs. (57)-(60)], we perform the integration by parts, and we assume that the arising integral over the surface of the integration volume is vanishing since $\mathcal{P} \rightarrow 0$ sufficiently fast as $\|\boldsymbol{\phi}\|,\left\|\boldsymbol{\phi}^{+}\right\| \rightarrow \infty$. However, as the experience with positive $P$ representation shows, this assumption quite often is wrong $[3,12,40]$.

In this section, we return to the simple scheme of Carusotto [13] and the constant trace scheme of Lacroix [20], and examine them for the presence of unaccounted boundary terms. First of all, we should check that the corresponding stochastic differential equations satisfy the conditions of existence and uniqueness of a solution.

Suppose we are given stochastic equation with a drift vector $\underline{\mathcal{A}}$ and a noise matrix $\underline{\mathcal{B}}$, which are continuous functions of $\phi$ and $\phi^{+}$. Then, there exists a solution to this stochastic equation, which is valid at all times $t \in[0, \infty$ ) (nonexploding), if the following restriction on the growth order is 
satisfied [26]:

$$
\left\|\underline{\mathcal{A}}\left[\underline{\phi}, \underline{\phi}^{+}\right]\right\|^{2}+\left\|\underline{\underline{\mathcal{B}}}\left[\underline{\phi}, \underline{\phi}^{+}\right]\right\|^{2} \leq K\left(1+\|\underline{\phi}\|^{2}+\left\|\underline{\phi}^{+}\right\|^{2}\right),
$$

where $K$ is some positive constant. Now, we look at the stochastic equation (105). In case of the simple scheme with $\kappa=\boldsymbol{\phi}, \boldsymbol{\kappa}^{+}=\boldsymbol{\phi}^{+}$, we obtain

$$
\begin{gathered}
\left\|\underline{\mathcal{A}}^{\Delta}\right\| \leq \frac{3 \sqrt{2}}{\sqrt{\hbar}}(N-1)\left\|Y_{ \pm}\right\|^{2}\|\boldsymbol{\phi}\|, \\
\left\|B_{ \pm}\right\| \leq \frac{1}{\sqrt{\hbar}}\left\|Y_{ \pm}\right\|\|\boldsymbol{\phi}\|,
\end{gathered}
$$

and so the condition (116) is met. In case of the constant trace scheme with $\boldsymbol{\kappa}=\boldsymbol{\phi}^{+}, \boldsymbol{\kappa}^{+}=\boldsymbol{\phi}$, we obtain

$$
\left\|\underline{\mathcal{A}}^{\Delta}\right\| \leq \frac{2 \sqrt{2}}{\sqrt{\hbar}}(N-1) \frac{\left\|Y_{ \pm}\right\|^{2}\|\boldsymbol{\phi}\|^{2}\left\|\boldsymbol{\phi}^{+}\right\|}{\left\langle\boldsymbol{\phi}^{+} \mid \boldsymbol{\phi}\right\rangle}
$$

We see that while the inner product $\left\langle\boldsymbol{\phi}^{+} \mid \boldsymbol{\phi}\right\rangle$ is being kept constant in the denominator, the numerator can grow as the power of the third degree. So, the conditions (116) are violated. Therefore, we expect that exploding trajectories (spikes) will appear during the simulation of the constant trace stochastic equations. As follows from the experience with positive $P$ representation $[3,12,40]$, the spiking behavior is usually accompanied by the appearance of the tails of the quasiprobability distribution, which falls off as a power law. If such tails are present, then the boundary terms do not vanish, and the stochastic method may give systematic discrepancies with the exact quantum dynamics, which do not reduce as we increase the sample size. Therefore, the constant trace scheme should be ruled out from the reliable stochastic simulation methods.

Next, we consider the condition of uniqueness of a solution to the stochastic differential equation, the generalized Lipshitz condition [26]: for every $p>0$ there should exist a constant $K_{p}$ such that

$$
\left\|\underline{\mathcal{A}}[\underline{\phi}]-\underline{\mathcal{A}}\left[\underline{\phi^{\prime}}\right]\right\|+\left\|\underline{\underline{\mathcal{B}}}[\underline{\phi}]-\underline{\underline{\mathcal{B}}}\left[\underline{\phi^{\prime}}\right]\right\| \leq K_{p}\left\|\underline{\phi}-\underline{\phi^{\prime}}\right\|
$$

for all times and all $\phi, \phi^{\prime}$ such that $\|\phi\| \leq p$ and $\left\|\phi^{\prime}\right\| \leq p$. The generalized Lipshitz condition (120) is satisfied by the simple scheme.

Now, let us turn to the problem of boundary terms in the simple scheme. Note that the drift evolution is unitary, and the noises $Q_{\phi, \phi} B_{+} d \boldsymbol{Z}$ and $Q_{\phi, \phi} B_{-} d Z^{*}$ are orthogonal to the vector $\phi$. Thus, the diffusion and the drift occur along the surfaces of concentric spheres, with centers at the origin $\boldsymbol{\phi}=0$. Applying the intuition of the classical geometry, one might think that there is no radial evolution at all, and the radial distribution function is constant. However, the Ito's calculus is more intricate. We consider the function $f=\langle\boldsymbol{\phi} \mid \boldsymbol{\phi}\rangle$, whose gradient is $\delta f / \delta \phi=\left(\boldsymbol{\phi}^{*}, \boldsymbol{\phi}\right)$, and the Hessian matrix is $\delta^{2} f / \delta \phi \delta \phi^{*}=I$, where $I$ is $2 \mathcal{N} \times 2 \mathcal{N}$ identity matrix. We substitute these results into the Ito's lemma (B17), and find that the terms with $\mathcal{A}$ cancel each other, and the noise terms vanish due to the orthogonality of diffusion. The resulting radial evolution is deterministic:

$$
\frac{d\langle\boldsymbol{\phi} \mid \boldsymbol{\phi}\rangle}{d t}=\frac{1}{2} \operatorname{Tr} \underline{\underline{\mathcal{D}}} .
$$

Since the noise matrices are bounded as (118), we have $\operatorname{Tr} \underline{\mathcal{D}} \leq 4\left\|Y_{ \pm}\right\|^{2}\|\boldsymbol{\phi}\|^{2} / \hbar$. Substituting this inequality into (121), we obtain

$$
\frac{d\langle\boldsymbol{\phi} \mid \boldsymbol{\phi}\rangle}{d t} \leq \frac{2}{\hbar}\left\|Y_{ \pm}\right\|^{2}\langle\boldsymbol{\phi} \mid \boldsymbol{\phi}\rangle .
$$

Integrating this inequality, we get that, if the initial quasiprobability is vanishing outside the ball of radius $R(0)$, then at any later time $t$ the quasiprobability is vanishing outside the ball of radius

$$
R(t) \leq R(0) \exp \left(t \frac{\left\|Y_{ \pm}\right\|^{2}}{\hbar}\right),
$$

and thus there is no problem of unaccounted boundary terms. We can always choose the initial distribution with finite $R(0)$ due to the diagonal representation (17).

The results of the treatment in this section are in agreement with the observations made in the works $[13,20]$. The constant trace scheme exhibits the spiking behavior [13,20] and the power-law tails of the radial distribution after the first spike time [13]. In the simple scheme, the spikes do not appear; since the width of the quasiprobability distribution is increasing exponentially with time, the variance of the results is experiencing the same growth [13]. However, since in the simple scheme the width of the quasiprobability distribution is always finite (provided so is the width of the initial distribution), there are no systematic errors (due to boundary terms), and the results of the simulation converge to the exact results provided we increase the size of the simulation sample [13].

\section{CONCLUSION}

We have studied the generalized phase-space representations underlying the stochastic wave-function methods using the example of Hartree-Fock dyadic method of Carusotto et al. $[13,28]$. We believe that we have been able to demonstrate the unifying strength of the phase-space approach. Various stochastic methods, derived from entirely different considerations in literature, can be obtained by a change of parameter in the general gauge transformation.

Analysis carried out in this paper leads to the following picture of how one can explore the possibilities for diffusive representations that are offered by a given operator ansatz. First of all, the situation is greatly simplified if the operator ansatz is an analytical function of its parameters. Then, the sufficient condition for the existence of diffusive stochastic master equations (for quantum system with pairwise interactions) is that the star-product operator correspondence for $\hat{\Psi}^{\dagger}(\mathbf{x}) \hat{\Psi}\left(\mathbf{x}^{\prime}\right)$ is a first-order differential operator. Next, we study the properties of the operator ansatz by finding the general form of its annihilators. The transposed annihilators provide us with a general form of all the gauge transformations of the quasiprobability master equations. If the operator ansatz is a homogeneous function of a finite degree, then there will be "absorption properties" which will allow us to transform between diffusion and drift, and between drift and potential terms. Now, we can optimize the master equations with respect 
to certain criteria or constraints. Having been assured that there exist stochastic equations eligible for simulation, we can investigate the properties of the quasiprobabilities. Such investigation can be accomplished with the aid of the Wick theorem for functional integration over the unit sphere. The overcompleteness of the operator ansatz can be checked, and the operator mappings for the density operator can be derived. If the operator ansatz has the form of a tensor product of two states, then there always will be diagonal and nondiagonal quasiprobability distributions. The operator mappings for the observables are obtained by evaluating the action of the transposed star-product representation of the observable on the trace of the operator ansatz.

The methods developed in this work can be applied to study the quasiprobability distributions which emerge in other stochastic methods, e.g., fermionic Hartree-Fock [14], Gutzwiller ansatz [15], etc.

\section{ACKNOWLEDGMENT}

This work is supported by the Russian Foundation for Basic Reseach (Grant No. 14-02-31109) and by the program of grants for postdocs of Saint Petersburg State University (Grant No. 11.50.1573.2013).

\section{APPENDIX A: MULTILINEAR FORMS AND INTEGRATION OVER THE FUNCTIONAL UNIT SPHERE}

In this Appendix, we review the properties of harmonic polynomials $[33,34]$ and evaluate the integral (9). A monomial of degree $p$ is a product of the form

$$
x_{1}^{a_{1}} \ldots x_{\mathcal{N}}^{a_{\mathcal{N}}} y_{1}^{b_{1}} \ldots y_{\mathcal{N}}^{b_{\mathcal{N}}}
$$

where the $a_{j}$ 's and $b_{j}$ 's are zero or positive integer powers, and their sum is equal to $p, \sum a_{j}+b_{j}=p$. Here, we consider $x_{j}$ and $y_{j}$ as the real and imaginary components of the complex variable $\phi_{j}$. If we change the variables $\left(x_{j}, y_{j}\right) \rightarrow\left(\phi_{j}, \phi_{j}^{*}\right)$, we come to the notion of the monomial of type $(r, s)$ :

$$
\phi_{1}^{* c_{1}} \ldots \phi_{\mathcal{N}}^{* c_{\mathcal{N}}} \phi_{1}^{d_{1}} \ldots \phi_{\mathcal{N}}^{d_{\mathcal{N}}}
$$

where $c_{j}$ 's and $d_{j}$ 's are zero or positive integer powers such that $\sum c_{j}=r$ and $\sum d_{j}=s$. We can group together all the monomials of the same type and consider the multilinear form of type $(r, s)$ :

$$
\begin{aligned}
\mathcal{P}_{(r, s)}\left(\boldsymbol{\phi}, \boldsymbol{\phi}^{*}\right) \\
=\prod_{j=1}^{r}\left\{\Delta V \sum_{k_{j}=1}^{\mathcal{N}} \phi_{k_{j}}^{*}\right\} \prod_{j=1}^{s}\left\{\Delta V \sum_{k_{j}^{\prime}=1}^{\mathcal{N}} \phi_{k_{j}^{\prime}}\right\} \\
\quad \times h\left(\mathbf{x}_{k_{1}}, \ldots, \mathbf{x}_{k_{r}} \mid \mathbf{x}_{k_{1}^{\prime}}, \ldots, \mathbf{x}_{k_{s}^{\prime}}\right) .
\end{aligned}
$$

Here, $h\left(\mathbf{x}_{k_{1}}, \ldots, \mathbf{x}_{k_{r}} \mid \mathbf{x}_{k_{1}^{\prime}}, \ldots, \mathbf{x}_{k_{s}^{\prime}}\right)$ is a tensor of type $(r, s)$ in the space $\mathbb{C}^{\mathcal{N}}$. Note that the expression (A3) does not change its value if we permute the left or the right indices of the tensor $h$. Hence, we assume that the tensor $h$ is symmetrical with respect to permutations of the indices of the same kind (left or right) without loss of generality. However, the tensor $h$ need not be either Hermitian or symmetrical with respect to permutations of indices of different kind. The multilinear form $\mathcal{P}_{(r, s)}\left(\boldsymbol{\phi}, \boldsymbol{\phi}^{*}\right)$
[Eq. (A3)] is a homogeneous polynomial of degree $r+s$ and hence we have for it the canonical decomposition [33,34]

$$
\mathcal{P}_{(r, s)}\left(\boldsymbol{\phi}, \boldsymbol{\phi}^{*}\right)=\sum_{j=0}^{\lfloor\min (r, s) / 2\rfloor}\left(\Delta V\|\boldsymbol{\phi}\|^{2}\right)^{j} \mathcal{P}_{(r-j, s-j)}^{(0)}\left(\boldsymbol{\phi}, \boldsymbol{\phi}^{*}\right),
$$

where $\mathcal{P}_{(r-j, s-j)}^{(0)}$ is a harmonic polynomial of order $r+s-$ $2 j$, satisfying the generalized Laplace's equation

$$
\Delta \mathcal{P}_{(r-j, s-j)}^{(0)}=4 \Delta V \sum_{k=1}^{\mathcal{N}} \frac{\delta^{2}}{\delta \phi_{k} \delta \phi_{k}^{*}} \mathcal{P}_{(r-j, s-j)}^{(0)}=0,
$$

where the symbol $\Delta$ is the generalized Laplace operator, and

$$
\frac{\delta}{\delta \phi_{k}}=\frac{1}{\Delta V} \frac{\partial}{\partial \phi_{k}}
$$

denotes the lattice counterpart of the variational derivative. The canonical decomposition (A4) is unique. Note the meaning of the $\Delta$ operator in (A5): it is a mapping which reduces the multilinear form type, $\Delta: \mathcal{P}_{(r, s)} \rightarrow \mathcal{P}_{(r-1, s-1)}^{\prime}$, where the tensors $h$ and $h^{\prime}$ of the forms $\mathcal{P}_{(r, s)}$ and $\mathcal{P}_{(r-1, s-1)}^{\prime}$ are related as

$$
\begin{aligned}
& h^{\prime}\left(\mathbf{x}_{k_{1}}, \ldots, \mathbf{x}_{k_{r-1}} \mid \mathbf{x}_{k_{1}^{\prime}}, \ldots, \mathbf{x}_{k_{s-1}^{\prime}}\right)=r s \Delta V \\
& \quad \times \sum_{l} h\left(\mathbf{x}_{l}, \mathbf{x}_{k_{1}}, \ldots, \mathbf{x}_{k_{r-1}} \mid \mathbf{x}_{l}, \mathbf{x}_{k_{1}^{\prime}}, \ldots, \mathbf{x}_{k_{s-1}^{\prime}}\right),
\end{aligned}
$$

i.e., the action of $\Delta$ is a sum of all the possible contractions of the form $\mathcal{P}_{(r, s)}$ over a single $\left(\phi^{*}, \phi\right)$ pair of sites. For brevity, we denote such an operation as a tensor trace $\operatorname{Tr}_{1}$, and the relation (A7) becomes $h^{\prime}=r s \operatorname{Tr}_{1} h$. Therefore, the canonical decomposition (A4) means that the multilinear form can be uniquely decomposed into the forms with traceless tensors $h^{(0)}: \operatorname{Tr}_{1} h^{(0)}=0$.

Denoting the integral over the unit sphere in (9) as the integral $\int d \Omega$ over the generalized solid angle, defined through the volume element

$$
\prod_{k} d \varphi_{k} d \varphi_{k}^{*}=\prod_{k} 2 d \varphi_{k}^{x} d \varphi_{k}^{y}=2^{\mathcal{N}}\|\boldsymbol{\phi}\|^{2 \mathcal{N}-1} d\|\boldsymbol{\phi}\| d \Omega,
$$

we have the following orthogonality relations for the harmonic multilinear forms [33]:

$$
\int d \Omega \mathcal{P}_{(r, s)}^{(0) *} \mathcal{P}_{\left(r^{\prime}, s^{\prime}\right)}^{(0)}=\text { const } \times \delta_{r r^{\prime}} \delta_{s s^{\prime}}
$$

Here, the harmonic form $\mathcal{P}_{(0,0)}^{(0)}$ of degree zero is just a complex constant. Due to the orthogonality (A9) and the decomposition (A4), we have for the integral of the multilinear form $\mathcal{P}_{(r, s)}$

$$
\int d \Omega \mathcal{P}_{(r, s)}=\delta_{r s} \mathcal{P}_{(0,0)}^{(0)} S_{2 \mathcal{N}-1} 2^{-\mathcal{N}},
$$

where $S_{2 \mathcal{N}-1}$ is the unit hypersphere volume (11). Using the harmonic projection formula [33], we can find $\mathcal{P}_{(0,0)}^{(0)}$ in the 
decomposition (A4):

$$
\mathcal{P}_{(0,0)}^{(0)}=\frac{(2 \mathcal{N}-2) ! !}{(2 s) ! !(2 \mathcal{N}+2 s-2) ! !} \Delta^{s} \mathcal{P}_{(s, s)}\left(\boldsymbol{\phi}, \boldsymbol{\phi}^{*}\right)
$$

Now, we interpret the action of the operator $\Delta^{s}$ as a sum over all the possible contractions between all the $\phi^{*}$ and $\phi$ sites, and finally by combining the results (A8), (A10), and (A11), we arrive at the Wick theorem (10) from Sec. II.

\section{APPENDIX B: FOKKER-PLANCK EQUATION IN COMPLEX VARIABLES}

In the generalized phase-space representations, the operator mappings are naturally expressed as functions of complex variables [2,4-7,12]. Therefore, the master equations for the quasiprobability distributions are usually the Fokker-Planck equations in complex variables. Here, we review the general form of the complex Fokker-Planck equation which corresponds to some stochastic differential equation. Using this general form, we can use the analyticity of the appropriate overcomplete basis in order to restore easily the necessary symmetries of the master equation in order to transform it into a genuine complex Fokker-Planck equation. In the context of positive $P$ function, this topic was considered in $[12,40]$, however, here for convenience of the reader we present a general and self-contained review.

\section{Complex Ito process with real noise}

Suppose we have the two set of variables $x_{j}$ and $y_{j}, j=$ $1, \ldots, n$. We consider the Ito stochastic process

$$
\begin{aligned}
& d x_{j}=A_{j}^{(x)} d t+\sum_{k=1}^{2 n} B_{j k}^{W(x)} d W_{k}, \\
& d y_{j}=A_{j}^{(y)} d t+\sum_{k=1}^{2 n} B_{j k}^{W(y)} d W_{k} .
\end{aligned}
$$

Here, the real Wiener increments $d W_{k}$ obey the conditions $\mathrm{E}\left[d W_{k}\right]=0$ and $\mathrm{E}\left[d W_{k} d W_{j}\right]=\delta_{k j} d t$. The time development of the probability distribution $\mathcal{P}(\boldsymbol{x}, \boldsymbol{y})$ for this process is described by the Fokker-Planck equation

$$
\begin{aligned}
\frac{\partial}{\partial t} \mathcal{P}= & -\sum_{j=1}^{n} \frac{\partial}{\partial x_{j}}\left\{A_{j}^{(x)} \mathcal{P}\right\}-\sum_{j=1}^{n} \frac{\partial}{\partial y_{j}}\left\{A_{j}^{(y)} \mathcal{P}\right\} \\
& +\frac{1}{2} \sum_{j=1}^{2 n} \sum_{k=1}^{2 n} \frac{\partial}{\partial \widetilde{x}_{j}} \frac{\partial}{\partial \widetilde{x}_{k}}\left\{D_{j k} \mathcal{P}\right\}
\end{aligned}
$$

where for brevity we have denoted $\tilde{\boldsymbol{x}}=\left(x_{1}, \ldots, x_{n}\right.$, $\left.y_{1}, \ldots, y_{n}\right)^{T}$. The diffusion matrix $D_{j k}$ is

$$
D=\left[\begin{array}{ll}
B^{W(x)}\left(B^{W(x)}\right)^{T} & B^{W(x)}\left(B^{W(y)}\right)^{T} \\
B^{W(y)}\left(B^{W(x)}\right)^{T} & B^{W(y)}\left(B^{W(y)}\right)^{T}
\end{array}\right] .
$$

Now, we change to the independent complex-conjugated variables $z_{j}=x_{j}+i y_{j}$ and $z_{j}^{*}, j=1, \ldots, n$. The corresponding complex Ito process driven by real noise is

$$
d z_{j}=A_{j} d t+\sum_{k=1}^{2 n} B_{j k}^{W} d W_{k}
$$

where $A_{j}=A_{j}^{(x)}+i A_{j}^{(y)}$ is an arbitrary complex vector, and $B_{j k}^{W}=B_{j k}^{W(x)}+i B_{j k}^{W(y)}$ is an arbitrary complex $n \times 2 n$ noise matrix. Now, we express Eq. (B3) in terms of $z_{j}$ and $z_{j}^{*}$. After some straightforward algebra, we have

$$
\begin{aligned}
\frac{\partial}{\partial t} \mathcal{P}= & -\sum_{j=1}^{n} \frac{\partial}{\partial z_{j}}\left\{A_{j} \mathcal{P}\right\}-\sum_{j=1}^{n} \frac{\partial}{\partial z_{j}^{*}}\left\{A_{j}^{*} \mathcal{P}\right\} \\
& +\frac{1}{2} \sum_{\alpha, j} \sum_{\beta, k} \frac{\partial}{\partial \underline{z}_{\alpha j}} \frac{\partial}{\partial \underline{z}_{\beta k}^{*}}\left\{\underline{\mathcal{D}}_{\alpha \beta j k} \mathcal{P}\right\},
\end{aligned}
$$

where for brevity we have introduced the vector $\underline{z}=\left(z, z^{*}\right)^{T}$ with $2 n$ components $\underline{z}_{1 k}=z_{k}$ and $\underline{z}_{2 k}=z_{k}^{*}$. The diffusion matrix $\underline{\underline{D}}_{\alpha \beta j k}$ becomes in this notation

$$
\begin{aligned}
\underline{\underline{\mathcal{D}}} & =\left[\begin{array}{cc}
B^{W} B^{W \dagger} & B^{W}\left(B^{W}\right)^{T} \\
{\left[B^{W}\left(B^{W}\right)^{T}\right]^{*}} & \left(B^{W} B^{W \dagger}\right)^{*}
\end{array}\right] \\
& =\left[\begin{array}{c}
B^{W} \\
B^{W *}
\end{array}\right]\left[\begin{array}{ll}
B^{W \dagger} & \left.\left(B^{W *}\right)^{\dagger}\right] .
\end{array}\right.
\end{aligned}
$$

We see that $\underline{\underline{D}}$ is Hermitian and positive (semi)definite. If we introduce the drift vector $\underline{\mathcal{A}}=\left(\boldsymbol{A}, \boldsymbol{A}^{*}\right)^{T}$ and the noise matrix $\underline{\mathcal{B}}_{\alpha j k}^{W}=\left[B_{j k}^{W}, B_{j k}^{W *}\right]^{T}$, then we conclude that for any complex Ito process $\underline{z}_{\alpha j}$ with real noise, given by the stochastic equation

$$
d \underline{z}_{\alpha j}=\underline{\mathcal{A}}_{\alpha j} d t+\sum_{k=1}^{2 n} \underline{\mathcal{B}}_{\alpha j k}^{W} d W_{k}
$$

the corresponding complex Fokker-Planck equation is

$$
\begin{aligned}
\frac{\partial}{\partial t} \mathcal{P}= & -\sum_{\alpha, j} \frac{\partial}{\partial \underline{z}_{\alpha j}}\left\{\underline{\mathcal{A}}_{\alpha j} \mathcal{P}\right\} \\
& +\frac{1}{2} \sum_{\alpha, j} \sum_{\beta, k} \frac{\partial}{\partial \underline{z}_{\alpha j}} \frac{\partial}{\partial \underline{z}_{\beta k}^{*}}\left\{\underline{\mathcal{D}}_{\alpha \beta j k} \mathcal{P}\right\} .
\end{aligned}
$$

Here, the diffusion matrix is related to the noise matrix as

$$
\underline{\underline{D}}_{\alpha \beta}=\underline{\mathcal{B}}_{\alpha}^{W}\left(\underline{\mathcal{B}}_{\beta}^{W}\right)^{\dagger}
$$

Now, suppose that we are given the master equation (ME) for some quasiprobability distribution, and that it should be made a genuine Fokker-Planck equation. The quasiprobability distribution is obtained through expansion over a certain analytical overcomplete basis. Then, in the ME only the terms with $\partial / \partial \underline{z}_{1 j}$ and $\partial^{2} / \partial \underline{z}_{1 j} \partial \underline{z}_{2 k}^{*}$ are fixed (they come from the operator phase-space mappings). The terms $\partial / \partial \underline{z}_{2 j}$, $\partial^{2} / \partial \underline{z}_{2 j} \partial \underline{z}_{\beta k}^{*}$, and $\partial^{2} / \partial \underline{z}_{\alpha j} \partial \underline{z}_{1 k}^{*}$ can be added at will due to the basis analyticity. Hence, we can always put the drift term into the canonical form (B8) just by adding to the ME the complex conjugates of the terms with $\partial / \partial \underline{z}_{1 j}$ (which are already present in ME). To put the diffusion matrix into the canonical form (B6) we have to look at the term

$$
\frac{1}{2} \sum_{j, k} \frac{\partial}{\partial \underline{z}_{1 j}} \frac{\partial}{\partial \underline{z}_{2 k}^{*}}\left\{\underline{\mathcal{D}}_{12 j k} \mathcal{P}\right\},
$$

which is already present in the ME. The matrix $\underline{\underline{D}}_{12 j k}$ should be symmetrical, that can be always achieved by employing 
the symmetry property of mixed derivatives. Then, the task of stochastic representation is reduced to the factorization

$$
\underline{\underline{D}}_{12}=B^{W}\left(B^{W}\right)^{T} \text {. }
$$

Having performed that, we immediately obtain that the underlying stochastic process is (B5) or, equivalently, is (B7).

We conclude this section by noting that the Ito's lemma for the stochastic process (B7) assumes the following form in the complex variables:

$$
\begin{aligned}
d f(\underline{z})= & \left\{\sum_{\alpha, j} \underline{\mathcal{A}}_{\alpha j} \frac{\partial}{\partial \underline{z}_{\alpha j}}+\frac{1}{2} \sum_{\alpha, \beta} \sum_{j, k} \underline{\mathcal{D}}_{\alpha \beta j k} \frac{\partial}{\partial \underline{z}_{\alpha j}} \frac{\partial}{\partial \underline{z}_{\beta k}^{*}}\right\} \\
& \times f(\underline{z}) d t+\sum_{\alpha} \sum_{j, k} \underline{\mathcal{B}}_{\alpha j k}^{W} \frac{\partial f(\underline{z})}{\partial \underline{z}_{\alpha j}} d W_{k},
\end{aligned}
$$

where $f(z)=f(\boldsymbol{x}, \boldsymbol{y})$ is an arbitrary twice-differentiable real or complex function.

\section{Complex Ito process with complex noise}

Sometimes when it is not evident how to perform the factorization (B11), it is helpful to consider the Ito process driven by complex noise. This is the case when the matrix $\underline{\mathcal{D}}_{12}$ is easily factorized into two different matrices, which are not the transposes of each other. The complex noise Ito process is obtained from the stochastic equation (B5) by splitting the Wiener increment vector $d \boldsymbol{W}$ into the two equal parts $d \boldsymbol{W}=$ $\left(d \boldsymbol{W}_{(x)}, d \boldsymbol{W}_{(y)}\right)^{T}$. Then, the complex Wiener increment is constructed as $d \boldsymbol{Z}=\left(d \boldsymbol{W}_{(x)}+i d \boldsymbol{W}_{(y)}\right) / \sqrt{2}$, and its statistical properties are $\mathrm{E}\left[d \underline{Z}_{\alpha j}\right]=0$ and $\mathrm{E}\left[d \underline{Z}_{\alpha j} d \underline{Z}_{\beta k}^{*}\right]=\delta_{\alpha, \beta} \delta_{j, k} d t$. The Ito process assumes the form

$$
d \underline{z}_{\alpha j}=\underline{\mathcal{A}}_{\alpha j} d t+\sum_{\beta, k} \underline{\underline{\mathcal{B}}}_{\alpha \beta j k}^{Z} d \underline{Z}_{\beta k},
$$

where it can be shown that the new noise matrix $\underline{\mathcal{B}}^{Z}$ is related to the the noise matrix $B^{W}$ from Eq. (B5) as

$$
\underline{\underline{\mathcal{B}}}^{Z}=\left[\begin{array}{ll}
B_{+} & B_{-} \\
B_{-}^{*} & B_{+}^{*}
\end{array}\right]=\underline{\mathcal{B}}^{W} U=\left[\begin{array}{c}
B^{W} \\
B^{W *}
\end{array}\right] U,
$$

where the unitary matrix $U$ is

$$
U=\frac{1}{\sqrt{2}}\left[\begin{array}{cc}
I & I \\
-i I & i I
\end{array}\right],
$$

and $I$ is the $n \times n$ identity matrix. Here, $B_{+}$and $B_{-}$are arbitrary complex $n \times n$ matrices now. We see from Eq. (B14) that the new noise matrix is related to the old one by a unitary transformation, and hence the stochastic process (B13) corresponds to the same Fokker-Planck equation (B8). Now, the only essential (i.e., fixed by phase-space mappings) term $\underline{\underline{D}}_{12}$ has the structure

$$
\underline{\underline{D}}_{12}=B_{+} B_{-}^{T}+\left(B_{+} B_{-}^{T}\right)^{T}
$$

We conclude this section by noting that the Ito's lemma for the stochastic process (B13) assumes the following form in the complex variables:

$$
\begin{aligned}
d f(\underline{z})= & \left\{\sum_{\alpha, j} \underline{\mathcal{A}}_{\alpha j} \frac{\partial}{\partial \underline{z}_{\alpha j}}+\frac{1}{2} \sum_{\alpha, \beta} \sum_{j, k} \underline{\underline{D}}_{\alpha \beta j k} \frac{\partial}{\partial \underline{z}_{\alpha j}} \frac{\partial}{\partial \underline{z}_{\beta k}^{*}}\right\} \\
& \times f(\underline{z}) d t+\sum_{\alpha, \beta} \sum_{j, k} \underline{\mathcal{B}}_{\alpha \beta j k}^{Z} \frac{\partial f(\underline{z})}{\partial \underline{z}_{\alpha j}} d \underline{Z}_{\beta k} .
\end{aligned}
$$

\section{Efficiency of stochastic representation}

Note that the representation (B16) is already quite general since we can always make the trivial choice $B_{+}=\underline{\mathcal{D}}_{12} / 2$ and $B_{-}=I$. So, why do we have to bother any more? The reason lies in the efficiency of the Monte Carlo simulation of the resulting stochastic differential equations. Suppose we evaluate the average values of a group of different observables during the simulation. We perform this by averaging over the random realizations of the trajectories in the generalized phase space. The efficiency of the Monte Carlo simulation is measured by the statistical errors of the averaged values. Therefore, the efficiency of the simulation is generally determined by the spread of the trajectories in the generalized phase space: the greater is the spread, the greater is the variance of the simulation results. The extreme case of the pure drift (no diffusion and no spread of the trajectories) has the $100 \%$ efficiency. Following the ideas of Carusotto et al. [13], we may devise a local measure $\sigma$ of the growth rate of the trajectory spread over each time interval $d t$. As such a measure $\sigma$, we can take

$$
\left.\sigma=\frac{1}{2} \operatorname{Tr} \underline{\underline{\mathcal{B}}}^{Z} \underline{\underline{\mathcal{B}}}^{Z}\right)^{\dagger}=\operatorname{Tr} \underline{\underline{\mathcal{D}}}_{11} .
$$

We see that the local efficiency of the simulation is not determined by the matrix $\underline{\underline{D}}_{12}$ directly (that comes from the phase-space mapping of the interaction term). The efficiency directly depends on how we factorize the term $\underline{\underline{D}}_{12}$. For example, in case of the complex noise factorization (B16),

$$
\sigma=\operatorname{Tr} B_{+} B_{+}^{\dagger}+\operatorname{Tr} B_{-} B_{-}^{\dagger},
$$

and for the trivial factorization described above we get

$$
\sigma=\frac{1}{4} \operatorname{Tr} \underline{\underline{D}}_{12}\left(\underline{\underline{D}}_{12}\right)^{\dagger}+n
$$

The number $n$ is the size of the grid upon which the wave functions are discretized. Therefore, the $n$ tends to be large. Since it turns out $[4,13]$ that the variance of the observable averages grows (very roughly) as the exponential of $\sigma t$ (or even faster), the trivial factorization is unacceptable. In fact, we can pose the variational problem: to find the matrices $B_{+}$ and $B_{-}$such that the $\sigma$ functional (B19) is minimal, under the constraint (B16).

\section{A test on nonpositive (semi)definitiness}

We finish this appendix by noting that sometimes it is useful to check that the diffusion matrix is not positive (semi)definite. In this case, the useful property is that the positive (semi)definite $\underline{\underline{D}}$ should satisfy the condition [41]

$$
\left|\underline{\underline{\mathcal{D}}}_{\alpha \beta j k}\right| \leq \sqrt{\underline{\underline{\mathcal{D}}}_{\alpha \alpha j j} \underline{\mathcal{D}}_{\beta \beta k k}} \leq \frac{1}{2}\left(\underline{\underline{D}}_{\alpha \alpha j j}+\underline{\underline{\mathcal{D}}}_{\beta \beta k k}\right) .
$$


[1] E. C. G. Sudarshan, Phys. Rev. Lett. 10, 277 (1963).

[2] P. D. Drummond and C. W. Gardiner, J. Phys. A: Math. Gen. 13, 2353 (1980).

[3] A. Gilchrist, C. W. Gardiner, and P. D. Drummond, Phys. Rev. A 55, 3014 (1997).

[4] P. Deuar and P. D. Drummond, J. Phys. A: Math. Gen. 39, 1163 (2006).

[5] J. F. Corney and P. D. Drummond, Phys. Rev. A 68, 063822 (2003).

[6] J. F. Corney and P. D. Drummond, Phys. Rev. B 73, 125112 (2006).

[7] J. F. Corney and P. D. Drummond, Phys. Rev. Lett. 93, 260401 (2004).

[8] R. Ng and E. S. Sørensen, J. Phys. A: Math. Theor. 44, 065305 (2011).

[9] P. Deuar and P. D. Drummond, Phys. Rev. Lett. 98, 120402 (2007).

[10] P. Deuar, J. Chwedenczuk, M. Trippenbach, and P. Zin, Phys. Rev. A 83, 063625 (2011).

[11] Q.-Y. He, M. D. Reid, B. Opanchuk, R. Polkinghorne, L. E. C. Rosales-Zárate, and P. D. Drummond, Front. Phys. 7, 16 (2012).

[12] P. Deuar and P. D. Drummond, Phys. Rev. A 66, 033812 (2002).

[13] I. Carusotto, Y. Castin, and J. Dalibard, Phys. Rev. A 63, 023606 (2001).

[14] O. Juillet and P. Chomaz, Phys. Rev. Lett. 88, 142503 (2002).

[15] I. Carusotto and Y. Castin, New J. Phys. 5, 91 (2003).

[16] J. Wilkie, Phys. Rev. E 67, 017102 (2003).

[17] L. Tessieri, J. Wilkie, and M. Çetinbaş, J. Phys. A: Math. Gen. 38, 943 (2005).

[18] A. Montina and Y. Castin, Phys. Rev. A 73, 013618 (2006).

[19] J. Wilkie, Y. M. Wong, and R. Ng, Chem. Phys. 369, 43 (2010).

[20] D. Lacroix, Ann. Phys. (NY) 322, 2055 (2007).

[21] D. Lacroix, Phys. Rev. A 72, 013805 (2005).

[22] H.-P. Breuer, Eur. Phys. J. D 29, 105 (2004).

[23] H.-P. Breuer, Phys. Rev. A 69, 022115 (2004).
[24] O. Juillet, F. Gulminelli, and P. Chomaz, Phys. Rev. Lett. 92, 160401 (2004).

[25] D. Lacroix and S. Ayik, Eur. Phys. J. A 50, 95 (2014).

[26] L. Arnold, Stochastic Differential Equations: Theory and Applications (Wiley, New York, 1974).

[27] H.-P. Breuer and F. Petruccione, The Theory of Open Quantum Systems (Oxford University Press, New York, 2007).

[28] I. Carusotto and Y. Castin, Ann. Henri Poincaré 4, S783 (2003).

[29] K. E. Cahill and R. J. Glauber, Phys. Rev. 177, 1857 (1969).

[30] K. E. Cahill and R. J. Glauber, Phys. Rev. 177, 1882 (1969).

[31] G. S. Agarwal and E. Wolf, Phys. Rev. D 2, 2161 (1970).

[32] G. S. Agarwal and E. Wolf, Phys. Rev. D 2, 2187 (1970).

[33] J. S. Avery, J. Comput. Appl. Math. 233, 1366 (2010).

[34] N. K. Vilenkin, Special Functions and the Theory of Group Representations (American Mathematical Society, Providence, RI, 1968).

[35] C. Gardiner, Stochastic Methods: A Handbook for the Natural and Social Sciences, Springer Series in Synergetics (Springer, Berlin, 2009).

[36] I. Carusotto and Y. Castin, J. Phys. B: At. Mol. Opt. Phys. 34, 4589 (2001).

[37] H. Reed and B. Simon, Methods of Modern Mathematical Physics I: Functional Analysis. Revised and Enlarged edition, Methods of Modern Mathematical Physics, Vol. 1 (Academic, London, 1980).

[38] M. Błaszak and Z. Domański, Ann. Phys. (NY) 327, 167 (2012).

[39] B. Simon, in Functional Integration and Quantum Physics, edited by S. Eilenberg and H. Bass, Pure and Applied Mathematics (Academic, New York, 1979).

[40] C. W. Gardiner and P. Zoller, Quantum Noise: A Handbook of Markovian and Non-Markovian Quantum Stochastic Methods with Applications to Quantum Optics, Springer Series in Synergetics (Springer, Berlin, 2004).

[41] R. A. Horn and C. R. Johnson, Matrix Analysis (Cambridge University Press, Cambridge, 1985). 